\title{
BlackCAT: A catalogue of stellar-mass black holes in X-ray transients $\star, \star \star$
}

\author{
J. M. Corral-Santana ${ }^{1}$, J. Casares ${ }^{2,3}$, T. Muñoz-Darias ${ }^{2,3}$, F. E. Bauer ${ }^{1,4,5}$, I. G. Martínez-Pais ${ }^{2,3}$, and D. M. Russell ${ }^{6}$ \\ ${ }^{1}$ Instituto de Astrofísica, Facultad de Física, Pontificia Universidad Católica de Chile (IA-PUC), Casilla 306, Santiago 22, Chile \\ e-mail: jcorral@astro.puc.cl \\ 2 Instituto de Astrofísica de Canarias (IAC), Vía Láctea, s/n, 38205 La Laguna, S/C de Tenerife, Spain \\ 3 Departamento de Astrofísica - Universidad de La Laguna (ULL), 38206 La Laguna, S/C de Tenerife, Spain \\ ${ }^{4}$ Millennium Institute of Astrophysics (MAS), Nuncio Monseñor Sótero Sanz 100, Providencia, Santiago Chile \\ 5 Space Science Institute, 4750 Walnut Street, Suite 205, Boulder, Colorado 80301, USA \\ 6 New York University Abu Dhabi, PO Box 129188, Abu Dhabi, UAE
}

Received 6 August 2015 / Accepted 24 October 2015

\begin{abstract}
Aims. During the last $\sim 50$ years, the population of black hole candidates in X-ray binaries has increased considerably, with 59 Galactic objects being detected in transient low-mass X-ray binaries, as well as a few in persistent systems (including $\sim 5$ extragalactic binaries). Methods. We collect near-infrared, optical, and X-ray information spread over hundreds of references to study the population of black holes in X-ray transients as a whole.

Results. We present the most updated catalogue of black hole transients. This contains X-ray, optical, and near-infrared observations, together with their astrometric and dynamical properties. The catalogue provides new and useful information in both statistical and observational parameters and provides a thorough and complete overview of the black hole population in the Milky Way. Analysing the distances and spatial distribution of the observed systems, we estimate a total population of $\sim 1300$ Galactic black hole transients. This means that we have only discovered less than $\sim 5 \%$ of the total Galactic distribution.
\end{abstract}

Key words. X-rays: binaries - stars: black holes - catalogs

\section{Introduction}

X-ray binaries (XRBs) are systems formed by either a neutron star (NS) or a black hole (BH), which is accreting mass from a companion donor star. Their detection began thanks to the development of space-based instrumentation in the 1960s, with the number of detections rising substantially since the implementation of all-sky monitors on board X-ray satellites, e.g. Ginga (1987-1991), RXTE (1996-2012) and, more recently, Swift (2004) and MAXI (2009). They are broadly divided into high-mass X-ray binaries (HMXBs) and low-mass X-ray binaries (LMXBs) according to the mass of the donor star. In the former, the early spectral type $(\mathrm{O}-\mathrm{B})$ massive $\operatorname{star}\left(\geq 10 M_{\odot}\right)$ mainly transfers material to the compact object through strong stellar winds. In LMXBs, on the other hand, the K-M spectral type star $\left(M_{2} \leq 1 M_{\odot}\right)$ fills the Roche lobe and transfers mass by Roche lobe overflow through the inner Lagrangian point (Charles \& Coe 2006). In this latter case, the material forms an accretion disc around the compact object whereby material is accreted. A few XRBs are also found with intermediate mass companions of spectral types in the range B-F. It has been proposed that these so-called intermediate-mass XRBs (IMXBs) may be the

\footnotetext{
* The complete version of this catalogue will be continuously updated at www.astro.puc.cl/BlackCAT and in the Virtual Observatory, including finding charts and data in other wavelengths.

$\star \star$ Tables A.1 to A.4 are also available in electronic form at the CDS via anonymous ftp to $(130.79 .128 .5)$ or via

http://cdsarc.u-strasbg.fr/viz-bin/qcat?J/A+A/587/A61
}

progenitors of some LMXBs through an episode of enhanced mass-transfer rate (Podsiadlowski et al. 2002).

The mass transfer rate $(\dot{M})$ largely determines the observational properties and gives rise to a subclassification within the LMXB class. Persistent sources are those with high accretion rates $\left(\dot{M} \sim 10^{-8} M_{\odot} \mathrm{yr}^{-1}\right.$; Tanaka \& Shibazaki 1996) and X-ray luminosities close to the Eddington limit. This high luminosity ensures that the outer parts of the accretion disc dominate the optical spectrum owing to reprocessing, and effectively hide the companion star. By contrast, transient sources are systems with low accretion rates $\left(\dot{M} \leq 10^{-9} M_{\odot} \mathrm{yr}^{-1}\right.$; Tanaka \& Shibazaki 1996) which exhibit long quiescent states and sporadic outburst episodes that are produced by thermal-viscous instabilities in the accretion disc (see Frank et al. 2002 for further explanation). During these outbursts, the brightness of the system rises to luminosities similar to those found in the persistent sources. After the outburst, transients decay back to quiescent states where they remain for most of their lifetimes. Typical recurrence times between outbursts span from years to centuries, depending on $\dot{M}$ (Ritter \& King 2002; McClintock \& Remillard 2006).

Observations have revealed that $\sim 25 \%$ of the transient LMXBs contain bursting NS (King et al. 1996a) while the rest $(\sim 75 \%)$ display X-ray spectral and/or timing properties that are characteristic of accreting black holes (hereafter we will refer to transient black hole systems as black hole transients or BHTs). In this paper we focus on the properties of Galactic BHTs since they represent the vast majority of the population of BHs. However, there are a few persistent, non-active, and extragalactic XRBs that harbour or may contain BHs. 


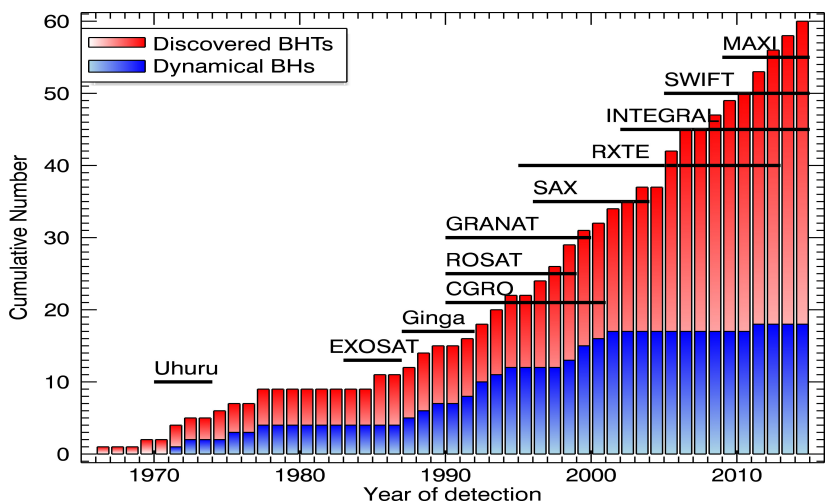

Fig. 1. Cumulative histogram of discovered (red) and dynamically confirmed (blue) BHTs as a function of time. Here, we also count Swift J1357.2-0933 as a dynamical BH. The lifetimes of the main X-ray satellites with all-sky monitor capabilities are shown with black lines.

Regarding the extragalactic population of BHs, dynamical evidence has been presented for LMC X-1 (a $11 \pm 1 M_{\odot} \mathrm{BH}$ with an O7III companion; Orosz et al. 2009) LMC X-3 (a $7.0 \pm 0.6 M_{\odot}$ BH with a B3-5V star; Orosz et al. 2014 and ValBaker et al. 2007) and M33 X-7 (a 16 $\pm 1 M_{\odot} \mathrm{BH}$ with a O7-8III star; Orosz et al. 2007) the first eclipsing stellar-mass BH ever detected.

On the other hand, indirect evidence for the presence of BHs in the HMXBs NGC 300 X-1 (12-24 $M_{\odot}$ BH with a Wolf-Rayet star, Crowther et al. 2010) and IC 10 X-1 (a 23-34 $M_{\odot}$ BH with a Wolf-Rayet star, Silverman \& Filippenko 2008) has been postulated. Owing to the unique challenges of observing the winddominated Wolf-Rayet companions, the masses of the compact objects in these systems are rather uncertain and, indeed, the presence of a neutron star cannot be ruled out (Binder et al. 2015; Laycock et al. 2015). Finally, we should also mention ultraluminous X-ray sources (ULXs), systems with X-ray luminosities that are greater than the Eddington limit for a $10 M_{\odot} \mathrm{BH}$. The source of these luminosities is still uncertain, and it has been proposed that they may be produced by intermediate mass BHs $\left(\sim 10^{3} M_{\odot}\right.$, see e.g. Miller et al. 2003) or stellar-mass BHs (e.g., Poutanen et al. 2007; Kawashima et al. 2012). More recently, for one case, it has been found that the compact object is a NS (M82 $\mathrm{X}-2$; Bachetti et al. 2014) which confounds our understanding of ULXs even further.

Hereafter, we will only focus on the Galactic population of BHs. We have performed a thorough search of all X-ray systems published in literature since 1962, when the first extrasolar X-ray source was detected, (the NS system Sco X-1, Giacconi et al. 1962) up until 2015. In Sect. 2 we motivate the generation of the catalogue with a historical view of the sample of BHTs and present the catalogue itself. In Sect. 3 we study the population of BHTs, where we analyse the vertical distribution of BHTs and constrain the expected number of systems in the Milky Way. In Sect. 4 we focus on the population of dynamically confirmed $\mathrm{BHs}$, presenting their distributions of periods, magnitudes, and masses.

\section{BlackCAT: the catalogue of Galactic BHs}

Since 1966 up to 2015, 59 BHTs have been discovered and are represented in Fig. 1 as a cumulative histogram of red bars, the slope of which represents a detection rate of $\sim 1.2$ targets per year. However, only 17 of these BHTs have been dynamically confirmed to harbour accreting BHs (i.e. mass function $\gtrsim 3 M_{\odot}$, see, e.g., Casares \& Jonker 2014) and are represented by the blue bars in Fig. 1. Here, we should also add Swift J1357.2-0933, where the dynamical confirmation is indirect because it is not based on the detection of the secondary star. However, there is robust evidence that it contains a BH (see Corral-Santana et al. 2013 and Mata Sánchez et al. 2015 for more details).

Therefore, the $17+1$ dynamical BHs represent $\sim 30 \%$ of the total number of BHTs discovered so far. This low fraction is due to most BHTs becoming too faint in quiescence for radial velocity studies using current instrumentation because of their intrinsically faint companions, high extinction, large distance, or a combination of the above. This problem could be alleviated with the exquisite sensitivity of future facilities like the European Extremely Large Telescope (E-ELT), especially with better IR instrumentation. The rest of the population of BHTs are named $\mathrm{BH}$ candidates because they share similar X-ray characteristics in outburst to the confirmed ones, but they lack a final dynamical confirmation (see, e.g. McClintock et al. 2006 and Belloni et al. 2011 for reviews on the X-ray observational properties of the $\mathrm{BH}$ candidates). Indeed, NS and BH sometimes display qualitatively similar phenomenology in outburst (see, e.g. Muñoz-Darias et al. 2014) and we cannot ignore that some of the objects included in this catalogue might harbour NS.

BlackCAT is a complete catalogue containing the astrometric, photometric (near-infrared -NIR-/optical magnitudes in outburst and quiescence), number of outbursts, the peak X-ray flux, distance, finding charts, and dynamical parameters of all the BHTs discovered so $\operatorname{far}^{1}$. In this paper we present the most relevant properties in the tables, as explained below.

We divide the catalogue into three main types of Galactic BHs: transients, persistent, and non-active, depending on their $\mathrm{X}$-ray activity.

\section{Persistent}

Cyg X-1 (a $15 \pm 1 M_{\odot}$ BH with a O9.7 Iab donor star, Orosz et al. 2011a) is the only confirmed Galactic BH in a persistent X-ray binary. On the other hand, 4U 1957+11 (Wijnands et al. 2002; Nowak et al. 2008, 2012; Hakala et al. 2014) and 1E 1740.72942 (Sunyaev et al. 1991) are persistent BH candidates, but they have not been dynamically confirmed. GRS 1758-258 (Mandrou 1990) is a quasi-persistent microquasar with a large extinction $A_{v} \sim 8.4$ (Mereghetti et al. 1997) that is located near the Galactic centre region. Finally, SS 443 (Stephenson \& Sanduleak 1977) is a non-transient source with a supercritical accretion regime onto a relativistic star (see Fabrika 2004 for a detailed review on the system). It is very likely a BH candidate with indirect arguments that support a compact object of $10-20 M_{\odot}$ with a high inclination (Eikenberry et al. 2001). However, despite it being intensively studied for almost 30 years, the nature of this system is still uncertain and it could be a Galactic ULX.

\section{Non-active BHs}

MWC 656 has recently been proposed as the first BH HMXB with a Be-type companion star (3.8-6.9 $M_{\odot}$ BH with a B1.5-2 III star, Casares et al. 2014). This is based on radial velocity curves of gas encircling the companion star and the spectroscopic mass of the Be star. This system has not shown any type of outbursting activity, so we label it as a non-active BH. Three other black hole

\footnotetext{
The electronic and most complete version of this catalogue is available on www astro.puc. cl/BlackCAT although all data will also be available through the Virtual Observatory.
} 
candidates have been discovered in globular clusters: the flatspectrum radio sources M22-VLA1 and M22-VLA2 (Strader et al. 2012) and M62-VLA1 (Chomiuk et al. 2013).

\section{Transients}

The remaining systems are transients, although some of them could be considered semi-persistents (e.g. GRS 1915+105 or 4U 1630-472) because of their long stays in the outburst state. Below, we detail the content of the tables presented in this paper. The systems included are either dynamically confirmed BHs or have shown spectra and/or timing features that are typically found in BHs (Belloni et al. 2011). We also note the existence of Cyg X-3 (Giacconi et al. 1967), a transient source showing strong radio outbursts. However, the nature of the primary, which accompanies the Wolf-Rayet donor, is unclear and it could be either an NS or a BH. Thus, we have excluded Cyg X-3 from our list of BHTs.

Table A.1 presents the basic astrometric properties of the BHTs sorted chronologically by year of detection in X-rays. The dynamically confirmed BHs are highlighted in grey. The column distribution is:

\section{(1) Year of discovery of the BHT;}

(2) Name of the system and optical counterpart when known;

(3-5) Right ascension (RA) and declination (DEC) coordinates in equinox J2000. The accuracy in the astrometry and the source of the coordinates are also shown;

(6-7) Galactic longitude $(\ell)$ and latitude $(b)$ in degrees;

(8-9) Estimated distance $(d)$ and height above the Galactic plane $(z)$ in kpc;

(10) Number of outbursts detected after discovery in X-rays;

(11) References for the detection, best coordinates, and distance determinations.

Table A. 2 shows the main properties, both in outburst and in quiescence, for all the BHTs presented in Table A.1. We also list the measured or predicted orbital period. Again, the dynamically confirmed BHs are highlighted in grey. The column distribution is:

(1) ID number used for cross-reference with the web version of this catalogue;

(2) Name of the system and optical counterpart when known;

(3) Peak X-ray flux in erg $\mathrm{s}^{-1} \mathrm{~cm}^{2}$, standardized to the $2-10 \mathrm{keV}$ band. To do so, we begin with the X-ray flux that has been published in the literature (or in archive). We assume a power-law spectrum with a photon index $\Gamma=2$ (Belloni et al. 2011) and the total neutral Galactic Hydrogen column density $\left(N_{\mathrm{H}}\right)$, published by Kalberla et al. (2005). If there is a measured $N_{\mathrm{H}}$ published in literature derived from direct $\mathrm{X}$-ray spectral analysis, we use this instead of the radio-derived interstellar one;

(4-5) Optical or IR magnitude in the peak of the outburst and quiescence, respectively, in the AB system. To document the original observed band, we provide the name of the band in its original system;

(6) Optical Galactic extinction $[E(B-V)]$ reported in the literature. If unknown, we list the total Galactic lineof-sight absorption given by the Schlafly \& Finkbeiner (2011) dust maps;

(7) Reported or estimated orbital period of the binary in hours;
(8) References for all the parameters above.

Table A.3 lists the optical/NIR photometry of the dynamical BHTs in quiescence:

(1) Preferred name of the dynamically confirmed BHT;

(2-9) Quiescent magnitudes of the dynamically confirmed BHTs. All the magnitudes were transformed to the AB system using the transformation coefficients taken from Table 2 in Frei \& Gunn (1994) and Eqs. (5) in Blanton et al. (2005). To document the original observed band, we provide the name in its original system;

(10) References of the magnitudes.

Table A.4 provides the dynamical parameters of the BHTs:

(1) Preferred name of the dynamically confirmed BHT;

(2) Spectral type of the companion star;

(3) The orbital period of the binary in hours;

(4) The radial velocity of the companion star $\left(K_{2}\right)$ in $\mathrm{km} \mathrm{s}^{-1}$;

(5) The mass function of the $\mathrm{BH} f\left(M_{1}\right)$ in $M_{\odot}$;

(6) The mass of the $\mathrm{BH}\left(M_{1}\right)$ in $M_{\odot}$. If there is an uncertain value, we prefer to show a range of masses;

(7) The binary mass ratio $q=M_{2} / M_{1}$;

(8) The inclination of the system $(i)$ in degrees;

(9) The rotational broadening $\left(v_{\text {rot }} \sin i\right)$ in $\mathrm{km} \mathrm{s}^{-1}$;

(10) References of all the parameters.

At the bottom of each table, we present a detailed list with the particularities marked in Table A.1 and Table A.2.

This census is the most updated available and, compared to previous catalogues (e.g. Gottwald et al. 1991; Chen et al. 1997; Remillard \& McClintock 2006; Ritter \& Kolb 2003; Liu et al. 2006, 2007), represents a substantial improvement in both statistics and observational parameters. While it only focuses on the population of BHs, it provides a thorough and more complete coverage of optical and NIR data and dynamical parameters ${ }^{2}$.

\section{Analysis of the spatial distribution of BHTs}

Table A.1 allows us to perform a statistical study of the distribution of BHTs in the Galaxy. In Fig. 2, we plot the 35 objects with estimated distances as viewed from the pole of the Milky Way. Filled orange circles represent the dynamically confirmed BHs while yellow stars mark the $\mathrm{BH}$ candidates. About $50 \%$ of the confirmed BHs are located within $4.5 \mathrm{kpc}$ of the Sun. This is a clear indication that interstellar extinction is a severe limitation to dynamical mass determinations. Moreover, from Fig. 2 it seems that almost all BHs lie within a spiral arm. Thus, for BHTs with uncertain distances, likely values could be estimated or constrained using the distance to the spiral arm.

Figure 3 shows the position of all the BHTs in Galactic coordinates overlaid onto a projected image of the Milky Way, as well as histograms of their distribution in Galactic longitude and latitude. There is a clear concentration of objects towards the direction of the bulge $\left(340^{\circ}<\ell<20^{\circ}\right.$ and $|b|<10^{\circ}$, Dwek et al. $1995)$ and disc. Only two objects are located at high Galactic latitudes (XTE J1118+480 at $b=+62^{\circ}$ and Swift J1357.2-0933 at $b=+50^{\circ}$ ), while three BHTs lie between $150^{\circ}<\ell<210^{\circ}$. Moreover, 31 out of the 59 BHTs have no reported quiescent

2 It is available at www.astro.puc.cl/BlackCAT and will be continuously updated with more systems and information in other spectral bands. 


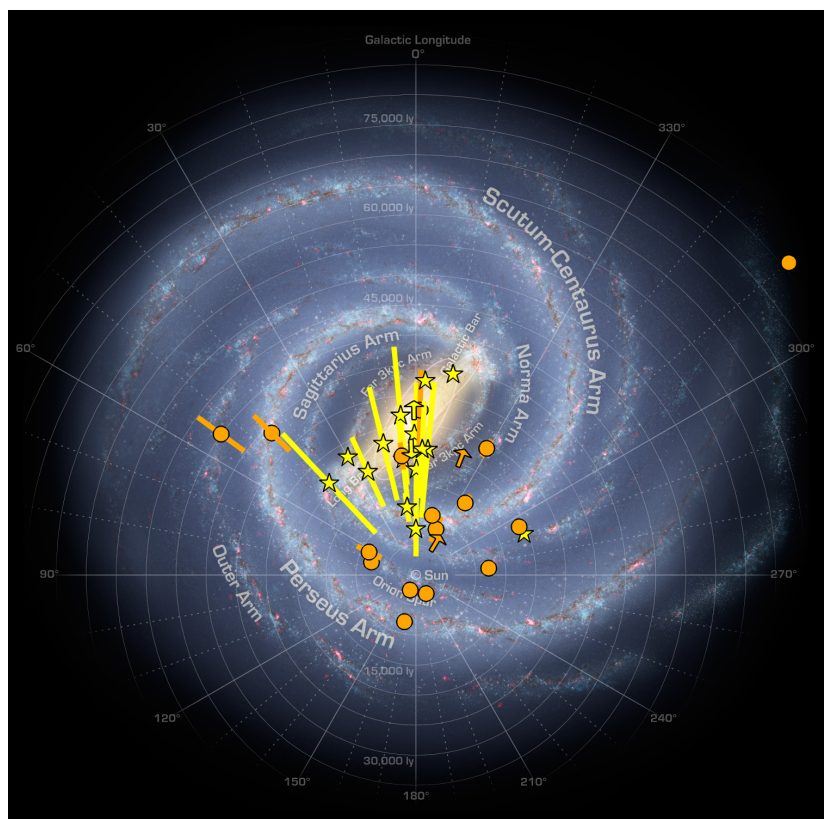

Fig. 2. Galactic distribution of 35 BHTs with distance estimates (Table A.1), as seen from the Galactic pole. Dynamically confirmed black holes are marked in orange circles, whereas $\mathrm{BH}$ candidates are indicated by yellow stars. Distance ranges are represented with bars while the systems with lower limits are indicated by arrows that follow the same colour index. Some systems are overlapped with other. Each faint white ring in the background image represents an additional $\sim 1.5 \mathrm{kpc}$ from the Sun. Objects seem to concentrate along the direction towards the Galactic centre. The dynamical BHT in the upper right of the figure is BW Cir. Background image credit: NASA/JPL-Caltech/R. Hurt (SSC/Caltech).

optical counterparts. In addition, 18 of them were detected in outburst, but they are either too faint in quiescence or the crowding of the field prevented the detection of a quiescent counterpart. On the other hand, 28 radio counterparts have been found (mostly during outburst). In Col. 5 of Table A.1, we indicate the range of the source of the coordinates together with the accuracy.

Stellar evolution predicts a population of $10^{8}-10^{9} \mathrm{BHs}$ in our Galaxy (van den Heuvel 2001; Remillard \& McClintock 2006). However, the total number of Galactic BHTs is very uncertain and depends on several considerations. Thus, van den Heuvel $(1992,2001)$ estimated a population between of 500 and 1000 BHTs, based on only three to four BHTs being known at the time. Current estimates predict $10^{3}-10^{4}$ Galactic BHTs (Romani 1998; Kiel \& Hurley 2006; Yungelson et al. 2006) based on models, although these numbers are probably an underestimate because of the existence of systems with extremely long outburst duty cycles (Ritter \& King 2002) or very faint peak X-ray luminosities (King \& Wijnands 2006).

Here we revisit the problem of the size of the Galactic population of BHs, based on the currently observed sample, which is heavily affected by extinction. Duerbeck (1984) modelled the density distribution of several interacting binaries in the Galaxy. In a similar way, we want to obtain the density of Galactic BHTs $\rho(z)$ and analyse the height distribution ( $z$; Col. 8 in Table A.1). Only 35 out of the 59 detected BHTs have estimated distances. Because of their transient nature, we have only detected those systems that went into outburst. Therefore, in a given period of time, we have only discovered a fraction of them (e.g. the brightest and/or closest BHTs - with the shortest outburst cycles). Thus, to derive the true $z$ distribution, we first need to find the maximum radial distance at which completeness of the sample is guaranteed, i.e. such that none of the X-ray outbursts are missed.

The completeness of the sample can be obtained from the analysis of the density of objects as a function of the radial distance that is projected onto the Galactic plane $(r)$. The latter is derived from the Galactic latitude and the distance listed in Cols. 5 and 6 of Table A.1.

Here, we have used 31 out of the 35 BHTs with a reported distance (we exclude the four systems with lower or upper limits). In addition, we consider a conservative $50 \%$ uncertainty in systems with only a rough estimate of distance. To derive the density of objects $(\Sigma)$ up to a radii $r$, we count the number of BHTs lying in cylinders or radius $r$ centred at the Sun and infinite height $(h)$. However, given the large uncertainties with distance, in some cases an object could be placed in more than a single cylinder within errors. To account for this effect, for each object we randomly generate 10000 values that assume a Gaussian distribution. Here, we naively assume the distance errors follow this sort of distribution, based on the assumption that all of the errors that went into the distance errors were Gaussian. In reality, we have no easy way of knowing this, as the values are from the literature, as determined by many groups, and in many cases are not fully documented. Thus, the final density of objects obtained in a cylinder is given by the median value of all the $10000 \Sigma$ that were obtained in the process, with the error given by the standard deviation. The result is shown in Fig. 4 where the density is represented as a function of the radial distance and normalized to its maximum value. In this figure we find a plateau in $\Sigma$ up to $r=4 \mathrm{kpc}$ (within errors) and a decrease for $r>4 \mathrm{kpc}$. We interpret this as an indication that the density of objects is approximately constant with increasing radii up to $\sim 4 \mathrm{kpc}$. We note here that it is difficult to establish a limit, but the density decreases clearly at $r>4 \mathrm{kpc}$, whereas it is not clear before this limit. Moreover, using the counting method proposed by Duerbeck (1984), we obtain a more abrupt decrease in $\Sigma$ for $r>4 \mathrm{kpc}$. However, we believe this technique is unsuitable for systems with large uncertainties in the distance.

We also perform a complementary analysis based on the X-ray luminosity. The least luminous BHT detected is XTE J1118+480 which reached an X-ray luminosity of $3.6 \times$ $10^{35} \mathrm{erg} \mathrm{s}^{-1}$ in the $2-10 \mathrm{keV}$ range (Dunn et al. 2010). Therefore, we can assume that no BHT peaked at a lower luminosity. The sensitivity limits by the all-sky monitor (ASM) on RXTE in the $2-10 \mathrm{keV}$ range have been above $2.4 \times 10^{-10} \mathrm{erg} \mathrm{s}^{-1} \mathrm{~cm}^{-2}$ since 1996, which means that any BHT that reached $3.6 \times 10^{35} \mathrm{erg} \mathrm{s}^{-1}$ within $3.5 \mathrm{kpc}$ would have been detected (without considering absorption). This is consistent with the method described above and, therefore, we assume that the sample of BHTs is complete out to $\sim 4 \mathrm{kpc}$.

There are ten objects in this volume (listed in Table 1). However, for the sake of the analysis we should only consider those BHTs detected since the rate of discoveries has become more or less constant. We assume that this has occurred since 1988 (see Fig. 1) when the sky started to be intensively scrutinized by all-sky monitors on-board X-ray satellites. Hence, we eliminate A0620-003 from this analysis because it was discovered in 1975 - before the beginning of the assumed constant rate of discoveries. This yields nine BHTs in the solar neighbourhood $(r \leq 4 \mathrm{kpc})$, discovered at a rate of $\sim 0.3 \mathrm{BHTs}^{-1}$ since 1988. For comparison, in the same time interval, $\sim 46$ BHTs have been discovered in the whole Galaxy (which implies a rate of $\simeq 1.7 \mathrm{BHTs}^{-1}$ ).

It is expected that the vertical distribution of BHTs will follow the same exponential function as the stellar distribution 


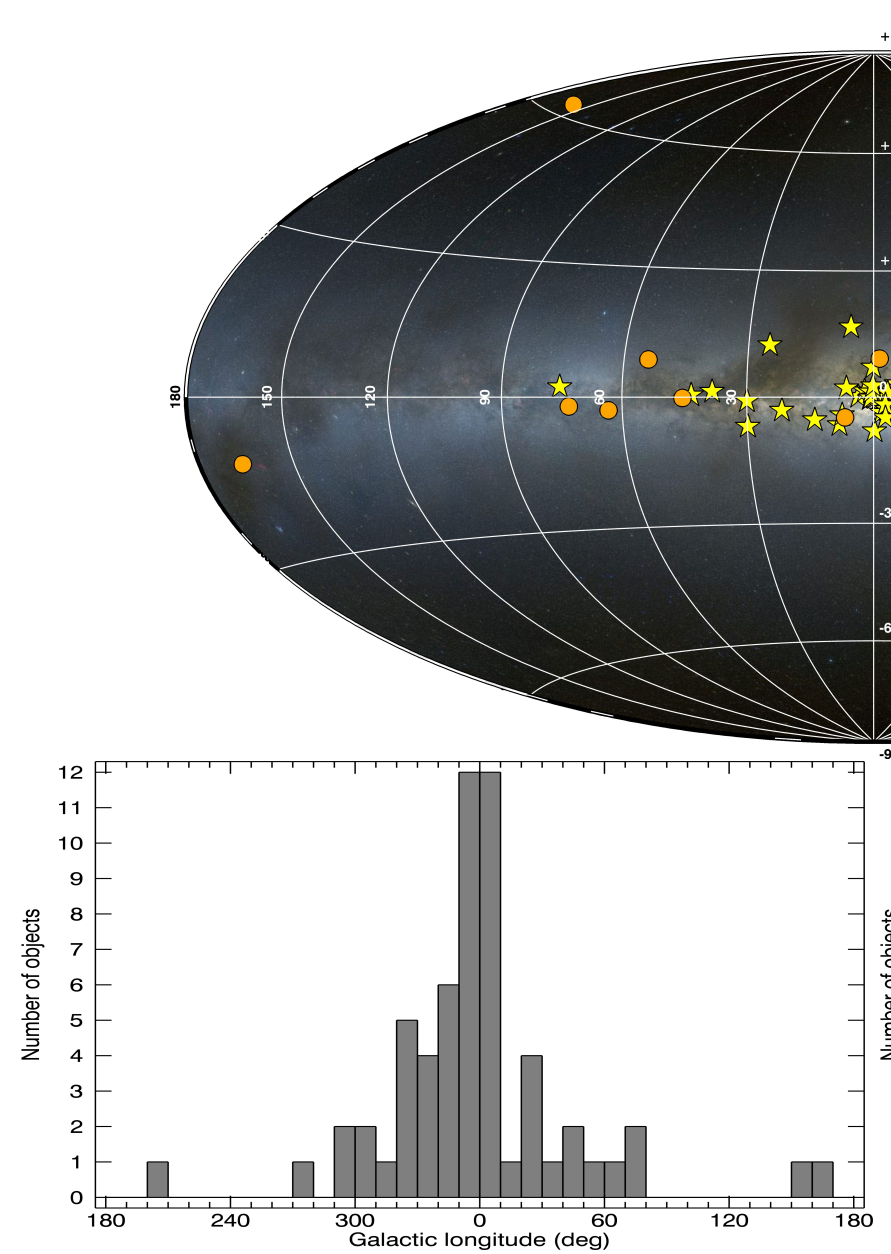

$+90 \quad \sum$ crandidates

ODynamicals

Fig. 3. Top: the distribution of dynamically confirmed BHs (circles) and BH candidates (stars) in the Galactic plane, using the Hammer projection. (Background image credit: Mellinger 2009). Some of the symbols are overlapped by others, especially in the Galactic centre region. Bottom: histogram of the distribution of $\mathrm{BH}$ transients in Galactic longitude (left) and latitude (right). A $10^{\circ}$ bin size in longitude and $5^{\circ}$ bin in latitude were used respectively.

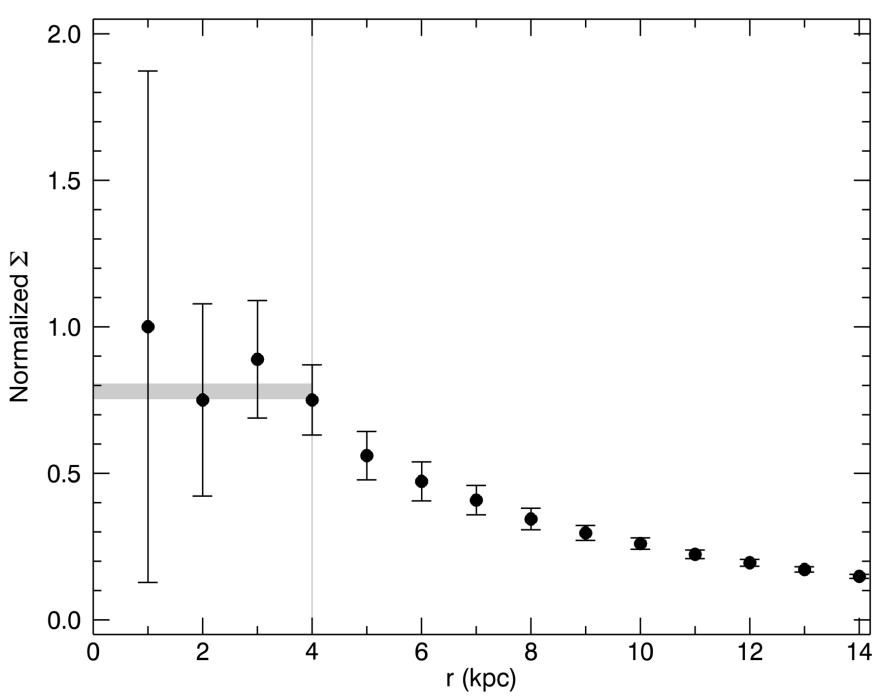

Fig. 4. Variation of the density of BHTs $(\Sigma)$ in a cylindrical volume of infinite height $h$ with increasing radii $r$. Above $r>4 \mathrm{kpc}$ the density decreases and, therefore, we infer the sample of BHTs is complete out to $r \sim 4 \mathrm{kpc}$ (grey bar).

(Duerbeck 1984). In addition, it is reasonable to assume that the population of BHTs is representative of the parent Galactic
Table 1. List of BHTs within $r=4 \mathrm{kpc}$ of completeness.

\begin{tabular}{llccl}
\hline \hline ID & Name & $\begin{array}{c}d \\
(\mathrm{kpc})\end{array}$ & $\begin{array}{c}z \\
(\mathrm{kpc})\end{array}$ & Ref. \\
\hline 40 & XTE J1818-245 & $3.55 \pm 0.75$ & $-0.26 \pm 0.05$ & Cadolle Bel et al. (2009) \\
33 & XTE J1650-500 & $2.6 \pm 0.7$ & $-0.16 \pm 0.04$ & Homan et al. (2006) \\
32 & XTE J1118+480 & $1.7 \pm 0.1$ & $1.52 \pm 0.09$ & Gelino et al. (2006) \\
21 & GRO J1655-40 & $3.2 \pm 0.2$ & $0.14 \pm 0.01$ & Hjellming \& Rupen (1995) \\
20 & GRS 1716-249 & $2.4 \pm 0.4$ & $0.29 \pm 0.05$ & della Valle et al. (1994) \\
19 & GRS 1009-45 & $3.8 \pm 0.3$ & $0.62 \pm 0.05$ & Gelino (2002) \\
17 & GRO J0422+32 & $2.5 \pm 0.3$ & $-0.51 \pm 0.06$ & Gelino \& Harrison (2003) \\
15 & GS 2023+338 & $2.4 \pm 0.1$ & $-0.09 \pm 0.01$ & Miller-Jones et al. (2009) \\
13 & GS 2000+251 & $2.7 \pm 0.7$ & $-0.14 \pm 0.04$ & Jonker \& Nelemans (2004) \\
\hline 7 & A0620-003 & $1.1 \pm 0.1$ & $-0.11 \pm 0.01$ & Cantrell et al. (2010) \\
\hline
\end{tabular}

population of BHTs, since there is no strong bias against their detection in X-rays (Özel et al. 2010).

Therefore, the space/time density distribution can be approximated by

$\rho^{*}(z)=\rho_{0}^{*} \exp \left(\frac{-|z|}{z_{0}}\right)\left(\mathrm{kpc}^{-3} \mathrm{yr}^{-1}\right)$

where $\rho_{0}^{*}$ is the space/time density of objects in the Galactic plane and $z_{0}$ the scale height of the distribution that is perpendicular to the plane of the Galaxy, both measured in the solar neighbourhood. 


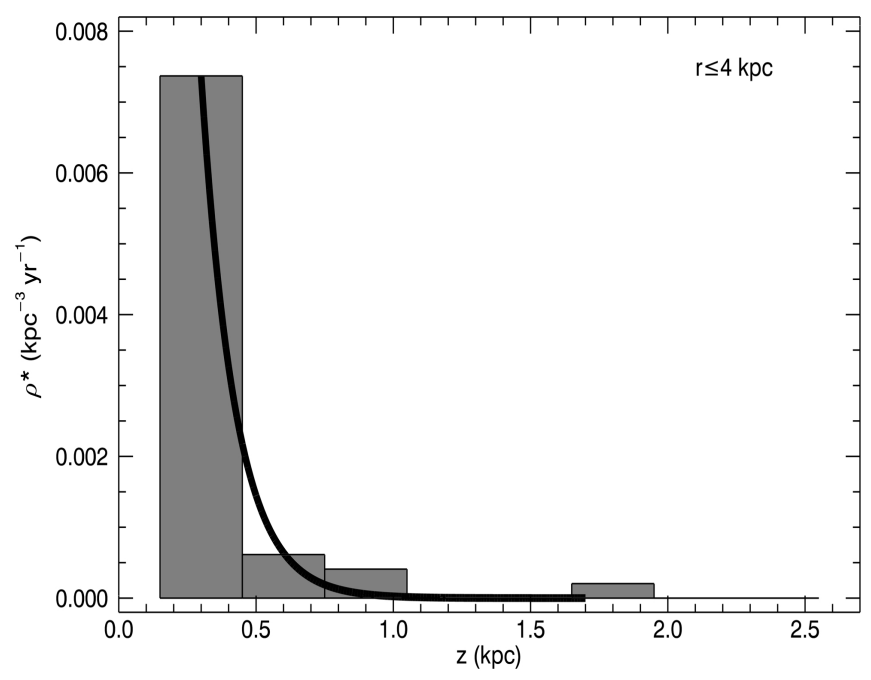

Fig. 5. Variation of the space/time density $\left(\rho^{*}\right)$ of BHTs with the scale above the Galactic plane $z$. The solid line represents the LevenbergMarquardt least-squares fit (Markwardt 2009) to the exponential function shown in Eq. (1).

Using a Levenberg-Marquardt non-linear least-squares fit (Markwardt 2009) of $\rho(z)$, we derive $\rho_{0}^{*}=0.08 \pm 0.01 \mathrm{kpc}^{-3} \mathrm{yr}^{-1}$ and $z_{0}=0.123 \pm 0.008 \mathrm{kpc}$ (see Fig. 5). This small scale height $z_{0}$ indicates a clear concentration of systems in the plane.

To obtain the final space density of BHTs $\rho(z)$, we must assume a mean outburst recurrence period (ORP). We have only observed transients for $\sim 50$ years and the observed ORPs for scrutinising the X-ray sky are thus biased by this limited time frame. In Fig. 6 we show the frequency of outbursts of all the BHTs over the past 50 years. The majority of systems have shown only one outburst, with only a small fraction showing multiple outbursts. The most extreme cases are H1743-322, GX 339-4, and 4U 1630-472, which have triggered 10, 19, and $\sim 20$ outbursts in $\simeq 50$ years (some of them were not considered "full outbursts"), respectively. In particular, 4U 1630-472 shows an ORP of 600-700 d, which lasted from 100-200 d up to 2.4 years (see, e.g. Kuulkers et al. 1997). There is also evidence for additional outbursts produced by A $0620+003$ (in 1917, Eachus et al. 1976) and V404 Cyg (in 1938 and 1956, Wachmann 1948; Richter 1989) detected by analysing photographic plates that were made previous to their discoveries. Nevertheless, we included only those outbursts discovered by X-ray satellites (i.e. since 1966) to be consistent and consequently, the observed ORP is biased by the $\simeq 50 \mathrm{yr}$ time lapse of X-ray astronomy observations. On the other hand, based on an analysis of the mass-transfer rate needed to reach the critical surface density that produces instabilities in the accretion disc, White \& van Paradijs (1996) consider it that BHTs have average ORPs above 100 yr. Therefore, the local density at the Galactic plane becomes $\rho_{0}=$ ORP $\rho_{0}^{*}=8 \pm 1\left(\frac{\mathrm{ORP}}{100 \mathrm{yr}}\right) \mathrm{kpc}^{-3}$.

A rough extrapolation of this local density distribution to the entire Galaxy, assuming no radial dependence, enables us to obtain the total number of BHTs by integrating Eq. (1) in cylindrical coordinates. Using $R=14 \mathrm{kpc}$ as the truncation radius for the Galactic disc and $H=2.5 \mathrm{kpc}$ as the maximum height above the Galactic plane given by MAXI J1659-152 (Kuulkers et al. 2013), we find a total number of $N=1280 \pm 120\left(\frac{\mathrm{ORP}}{100 \mathrm{yr}}\right)$ BHTs in the Galaxy with comparable properties to the systems detected so far, i.e. with peak X-ray luminosities a few tenths of the Eddington luminosity. This is consistent with the results

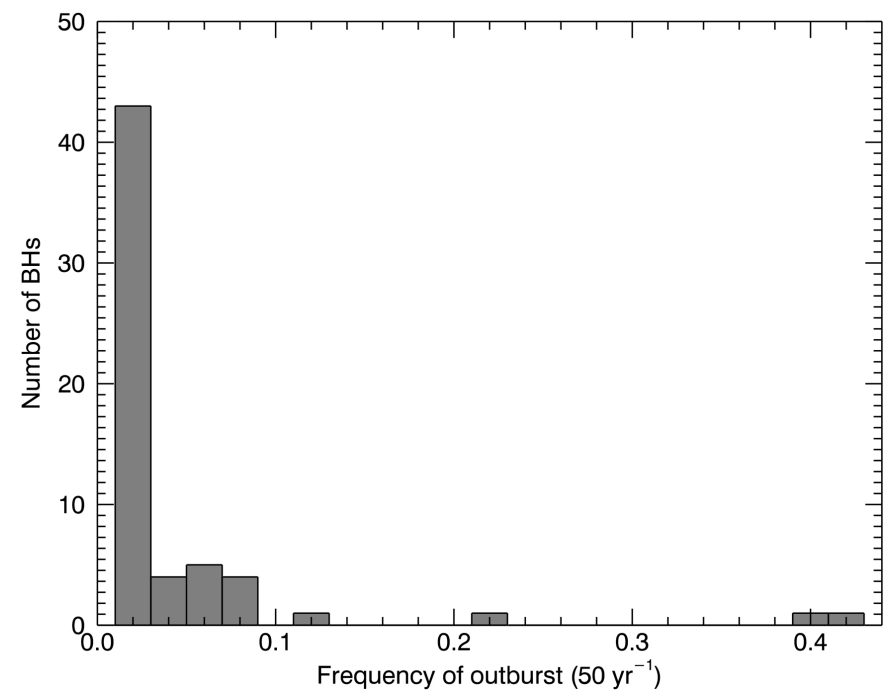

Fig. 6. Histogram of the frequency of outbursts detected over 50-yr period for all BHTs.

obtained using other techniques (van den Heuvel 1992; Tanaka 1992; White \& van Paradijs 1996; Romani 1998) and implies that only $\sim 4 \%$ of Galactic BHTs have been discovered.

Our empirical estimate is an order of magnitude lower than the $10^{4}$ BHTs predicted by Kiel \& Hurley (2006) or Yungelson et al. (2006) using population synthesis models. However, we note that our analysis is based on the study of observed systems but limited only to the nine BHTs with reliable distance estimates, which are located in a cylinder of $4 \mathrm{kpc}$ radius centred on the Sun. In addition, we have assumed that the solar vertical distribution $\left(\rho_{0}, z_{0}\right)$ can be extrapolated to other regions of the Galaxy. However, the bulge contains $\sim 30 \%$ of the stellar mass of the Galaxy, which is confined in a reduced spheroid and it is expected to host a higher concentration of BHTs (Muno et al. 2005). Furthermore, we considered a cylinder with a height defined by MAXIJ1659-152 (the object with the highest $h$ ) but there could be objects located at higher distances over the plane. Finally, we normalized our estimated value to an average recurrence period of $100 \mathrm{yr}$, which explicitly does not take into consideration any systems with lower accretion rates or longer recurrence periods, nor does it account for a likely population of intrinsically faint X-ray BHTs. Taking in all of the above, we conclude that our crude calculation of the number of BHTs expected in the Galaxy is very conservative and sets a lower limit to the hidden population.

\section{Physical properties of dynamical BHTs}

With the information on the apparent quiescent magnitudes, distances, and reddening listed in Tables A.1-A.4, we can recover the $R$-band absolute magnitudes (corrected for extinction and in the AB system) of the BHTs in quiescence. The magnitude distribution (Fig. 7, left) peaks strongly at $M_{R} \simeq 4-6$, where $\sim 40 \%$ of the systems lie. This result is expected since the quiescent spectra are mainly dominated by the light from the donor (mostly K-type stars) with some contribution from the accretion flow (typically $\leq 50 \%$ in this waveband). From Table A.4, we note a small fraction of IMXBs with A-F spectral-type companions (V4641 Sgr, 4U J1543-475, and GRO J1655-40), which have been indicated in black in Fig. 7.

In addition, from Table A. 2 we can revisit the observed distribution of orbital periods that shows a bimodal shape with a 

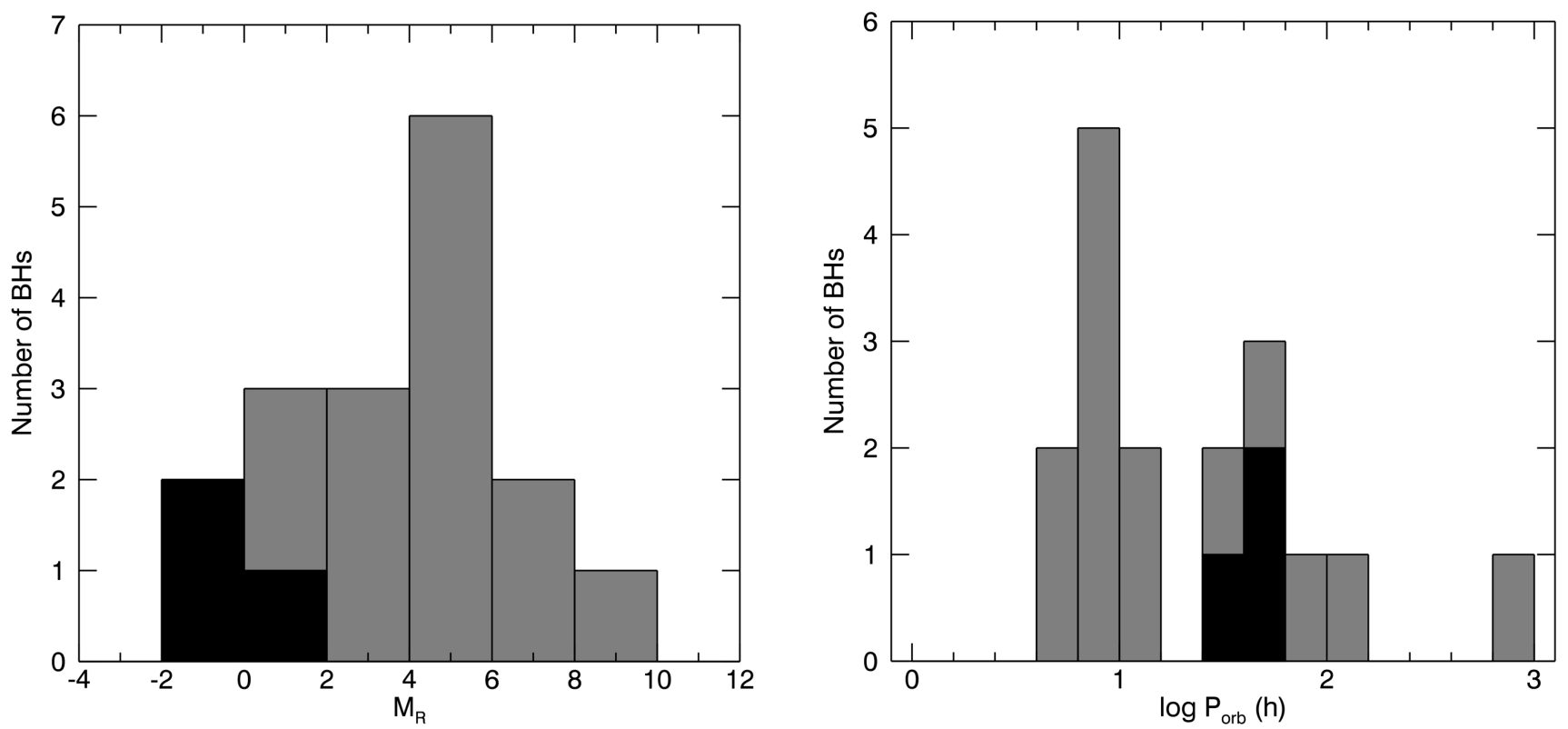

Fig. 7. Histograms of the 17 dynamically confirmed BHs. Left: extinction-corrected absolute $R$-band magnitudes in bins of 2 mag. The black histogram denotes confirmed IMXBs. Right: orbital periods in a logarithmic scale using bins of 0.2 .

gap at $\sim 15 \mathrm{~h}$, known as the bifurcation period (Fig. 7 , right). This is driven by different evolutionary paths, with systems above the gap evolving towards longer orbital periods through nuclear evolution of the donor stars, while angular momentum losses shrink the orbit of the binaries below the gap (Pylyser \& Savonije 1988; Menou et al. 1999). In this case, the IMXBs are located at long orbital periods, consistent with the presence of giant/subgiant donor stars. The majority of systems have periods between $6-10 \mathrm{~h}$, which corresponds to main-sequence or slightly evolved K-type donors filling their Roche lobes.

It is noticeable that none of the 59 BHTs listed in Tables A.1 and A.2 present X-ray or optical eclipses (the extragalactic BHHMXB M33 X-7 is the only one known as showing eclipses). In fact, all the dynamical BHTs found so far have binary inclinations $\lesssim 75^{\circ}$ (see Table A.4). However, it is expected that at least $20 \%$ of the 17 dynamically confirmed BHs will have $i>75^{\circ}$, taking an isotropic distribution of inclinations into consideration. Therefore, our estimate of the expected distribution of BHTs may be underestimated by $20 \%$ and should be $N \sim 1600\left(\frac{\mathrm{ORP}}{100 \mathrm{yr}}\right)$, although this percentage is probably smaller than the uncertainty associated with our systematic errors. Narayan \& McClintock (2005) propose that the lack of high inclination systems is due to a selection effect, which is produced by the inner accretion disc hiding or obscuring the central $\mathrm{BH}$, making these systems very faint in X-rays and preventing their detection during outbursts. For typical disc-flaring angles of $\sim 12^{\circ}$ (de Jong et al. 1996), the outer disc rim will obscure the central X-ray source permanently when viewed at inclinations $\gtrsim 78^{\circ}$.

Swift J1357.2-0933 may be the first object to be seen edgeon according to the optical properties that were displayed during the decay from its 2011 outburst (Corral-Santana et al. 2013), but it did not show either X-ray or optical eclipses. Armas Padilla et al. (2014) and Torres et al. (2015) present alternative explanations to the high inclination that is based on the quiescent X-ray properties and the Hydrogen column density obtained from the $\mathrm{Na}$ doublet in outburst, respectively. However, Mata Sánchez et al. (2015) present new evidencet that supports the edge-on configuration.

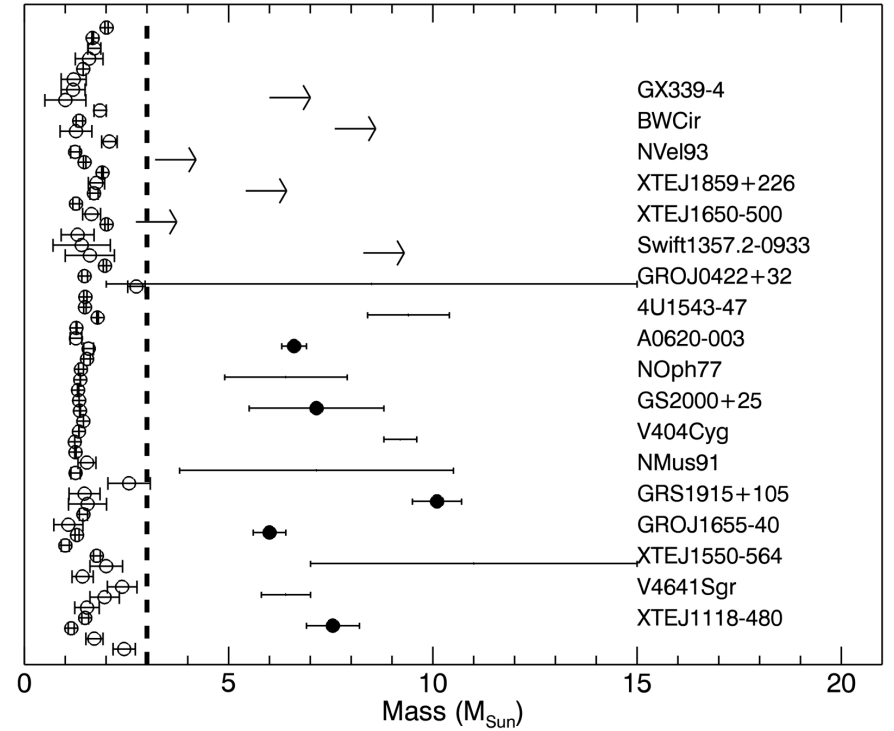

Fig. 8. Distribution of observed compact object masses. The vertical dashed line represents the maximum mass allowed for NS (Fryer \& Kalogera 2001). Open circles below that limit represent the masses of the NS compiled by Lattimer \& Prakash (2005), extended with updated data from Özel et al. (2012) and Antoniadis et al. (2013). The solid circles indicate reliable $\mathrm{BH}$ masses (adopting the values favoured by Casares \& Jonker 2014), while arrows indicate lower limits based on mass functions and upper limits to the inclination.

An accurate determination of the binary inclination is crucial for obtaining the mass of the compact object. The mass distribution of black holes has a significant impact on the physics of supernova explosions, the survival of interacting close binaries and the equation of state of nuclear matter. The distribution of masses of compact objects is expected to be smooth because of its correlation with the distribution of their progenitor masses (Fryer \& Kalogera 2001). However, the observed distribution shows a gap between neutron stars and BHs between $2-5 M_{\odot}$ (Fig. 8). Unlike Özel et al. (2010, 2012), here we only show with solid 
circles those systems with the most reliable mass determinations following Casares \& Jonker (2014). On the contrary, for those systems with inaccurate masses, we prefer to display large bars which encompass both the minimum and maximum published masses.

The existence of this mass gap is under debate. Özel et al. (2010) argue that selection effects may bias the observed distribution at high $\mathrm{BH}$ masses $\left(\geq 10 M_{\odot}\right)$ but cannot explain the gap that may be related to the physics of supernova explosions. Farr et al. (2011) perform a Bayesian analysis of the BH mass distribution and conclude that their larger sample provides strong evidence for the existence of the gap, reinforcing the results by Bailyn et al. (1998) and Özel et al. (2010). However, Kreidberg et al. (2012) warn about a large source of systematic errors that arise from a possible underestimation of the inclination angle $i$. This is produced by assuming that the emission from the accretion disc (i.e. the disc veiling) is negligible in the infrared when performing ellipsoidal modelling. They found that, in the case of A0620-003, this led to an underestimate of the inclination angle of at least $10^{\circ}$. Because of the cubic dependence of the mass function with $\sin i$, this could lead to a considerable overestimate of the BH mass. Correcting the BH mass in GRO J0422+32 from the estimated bias in the inclination, Kreidberg et al. (2012) find that the BH would lie in the gap. However, they also note that if this object is excluded from the analysis, previous conclusions remain intact.

On the other hand, Belczynski et al. (2012) and Fryer et al. (2012) state that the mass gap may be real and it could reveal new insights into the supernovae explosion models. More recently, Kochanek (2014) suggest an alternative explanation that is based on the absence of red supergiants in the range 16.5-25 $M_{\odot}$ as progenitors of type IIp supernova. As a result of the weakly bound hydrogen envelopes on these massive stars, they eject the outer layers, which leaves a BH with the mass of the star's helium core $\left(5-8 M_{\odot}\right)$. This would explain (i) the lack of supernova progenitors in the $16-25 M_{\odot}$ mass range and (ii) the existence of the mass gap and the typical masses of BHs.

\section{Conclusions}

We have presented the main properties of a large catalogue of BHTs. Of the 59 BHTs detected in outburst so far, only $\sim 30 \%$ have been confirmed as dynamical BHTs. A number of them (35) have distance estimates that allow us to study the Galactic distribution. This in turn results in a population of $\sim 1280\left(\frac{\mathrm{ORP}}{100 \mathrm{yr}}\right)$ such systems in the Milky Way. This value agrees with previous estimates that use other techniques but is an order of magnitude lower than theoretical predictions, which are based on population synthesis models. We argue that this value must be considered as a lower limit since it is based on the extrapolation of a small number of systems (nine out of the 59 systems detected so far).

We provide several tables listing the astrometric parameters, distances, number of eruptions, X-ray fluxes at the peak of the outburst and an outburst and a quiescence magnitude, reddening, and orbital periods. For dynamically confirmed systems, we have also detailed the magnitudes in quiescence in every available optical and NIR band, together with their dynamical parameters. In the online version of the catalogue, we have also added finding charts, links to the references and relevant information for forthcoming observations of these systems. We plan to include more information on other wavelengths in the near future (e.g. radio or X-ray states) and update the online catalogue with new targets once discovered.

Acknowledgements. We thank the anonymous referee for useful comments. We acknowledge financial support from CONICYT-Chile grants FONDECYT Postdoctoral Fellowship 3140310 (JMC-S), FONDECYT 1141218 (FEB), Basal-CATA PFB-06/2007 (JMS-C, FEB), "EMBIGGEN" Anillo ACT1101 (FEB), the Ministry of Economy, Development, and Tourism's Millennium Science Initiative through grant IC120009, awarded to The Millennium Institute of Astrophysics, MAS (FEB) and the Spanish Ministerio de Economía y Competitividad (MINECO) under grant AYA 2013-42627 (JC, TMD, IGMP). T.M.D. acknowledges hospitality during his 2015 visit to IA-PUC. This work makes use of observations from the LCOGT network, the CNTAC programs ID CN2014B-44 and CN2015A-88, the ESO Science Archive Facility under request number jcorral-160882, the GTC Public Archive at CAB (INTA-CSIC), and the Isaac Newton Group archive, which is maintained as part as the CASU Astronomical Data Centre at the Institute of Astronomy, Cambridge and the LCOGT Archive, which is operated by the California Institute of Technology, under contract with the Las Cumbres Observatory. We are thankful to Danny Steeghs and Manuel A. P. Torres for providing us with some of the finding charts and Jorge Andrés Perez Prieto for his help with the creation of the web. We have used the web applications of the FTOOLS (Blackburn 1995) and PIMMS (Mukai 1993) software to create the transformation of the X-ray fluxes. Some peak X-ray fluxes were provided by the ASM/RXTE teams at MIT and at the RXTE SOF and GOF at NASA's GSFC. This research made use of the MAXI data provided by RIKEN, JAXA and the MAXI team (Matsuoka et al. 2009).

\section{References}

Altamirano, D., Belloni, T., Linares, M., et al. 2011, ApJ, 742, L17 Altamirano, D., Wijnands, R., Heinke, C. O., \& Bahramian, A. 2014, ATel, 6469, 1

Angelini, L., \& White, N. E. 2003, ApJ, 586, L71

Antoniadis, J., Freire, P. C. C., Wex, N., et al. 2013, Science, 340, 448

Armas Padilla, M., Degenaar, N., Russell, D. M., \& Wijnands, R. 2013, MNRAS, 428, 3083

Armas Padilla, M., Wijnands, R., Degenaar, N., et al. 2014, MNRAS, 444, 902 Augusteijn, T., Coe, M., \& Groot, P. 2001a, IAU Circ., 7710, 2

Augusteijn, T., Kuulkers, E., \& van Kerkwijk, M. H. 2001b, A\&A, 375, 447

Bachetti, M., Harrison, F. A., Walton, D. J., et al. 2014, Nature, 514, 202

Bailyn, C. D., Orosz, J. A., Girard, T. M., et al. 1995, Nature, 374, 701

Bailyn, C. D., Jain, R. K., Coppi, P., \& Orosz, J. A. 1998, ApJ, 499, 367

Ballet, J., Denis, M., Gilfanov, M., et al. 1993, IAU Circ., 5874, 1

Barret, D., Roques, J. P., Mandrou, P., et al. 1992, ApJ, 392, L19

Barthelmy, S. D., D’Ai, A., D’Avanzo, P., et al. 2015, GCN Circ., 17929, 1

Beekman, G., Shahbaz, T., Naylor, T., \& Charles, P. A. 1996, MNRAS, 281, L1

Beekman, G., Shahbaz, T., Naylor, T., et al. 1997, MNRAS, 290, 303

Beer, M. E., \& Podsiadlowski, P. 2002, MNRAS, 331, 351

Belczynski, K., Wiktorowicz, G., Fryer, C. L., Holz, D. E., \& Kalogera, V. 2012, ApJ, 757, 91

Belloni, T., Colombo, A. P., Homan, J., Campana, S., \& van der Klis, M. 2002, A\&A, 390, 199

Belloni, T. M., Motta, S. E., \& Muñoz-Darias, T. 2011, BASI, 39, 409

Binder, B., Gross, J., Williams, B. F., \& Simons, D. 2015, MNRAS, 451, 4471

Blackburn, J. K. 1995, in Astronomical Data Analysis Software and Systems IV, eds. R. A. Shaw, H. E. Payne, \& J. J. E. Hayes, ASP Conf. Ser., 77, 367

Blanton, M. R., Schlegel, D. J., Strauss, M. A., et al. 2005, AJ, 129, 2562

Boeer, M., Greiner, J., \& Motch, C. 1996, A\&A, 305, 835

Borozdin, K., Sunyaev, R., \& Arefiev, V. 1993, IAU Circ., 5878, 1

Borozdin, K., Alexandrovich, N., Arefiev, V., et al. 1994, IAU Circ., 6083, 1

Borozdin, K. N., Aleksandrovich, N. L., Aref'ev, V. A., Syunyaev, R. A., \& Skinner, G. K. 1995, Astron. Lett., 21, 212

Bradt, H. V. D., \& McClintock, J. E. 1983, ARA\&A, 21, 13

Brandt, S., Castro-Tirado, A. J., Lund, N., et al. 1992, A\&A, 254, L39

Brocksopp, C., Jonker, P. G., Fender, R. P., et al. 2001, MNRAS, 323, 517

Brocksopp, C., McGowan, K. E., Krimm, H., et al. 2006, MNRAS, 365, 1203

Brocksopp, C., Jonker, P. G., Maitra, D., et al. 2010, MNRAS, 404, 908

Buxton, M. M., \& Bailyn, C. D. 2004, ApJ, 615, 880

Buxton, M., Sood, R., Rayner, D., et al. 1998, IAU Circ., 6827, 1

Buxton, M. M., Bailyn, C. D., Capelo, H. L., et al. 2012, AJ, 143, 130

Cadolle Bel, M., Ribó, M., Rodriguez, J., et al. 2007, ApJ, 659, 549

Cadolle Bel, M., Prat, L., Rodriguez, J., et al. 2009, A\&A, 501, 1

Callanan, P. J., \& Charles, P. A. 1991, MNRAS, 249, 573

Callanan, P. J., McCarthy, J. F., \& Garcia, M. R. 2000, A\&A, 355, 1049

Calvelo, D. E., Tzioumis, T., Corbel, S., Brocksopp, C., \& Fender, R. P. 2009a, ATel, 2135, 1

Calvelo, D. E., Vrtilek, S. D., Steeghs, D., et al. 2009b, MNRAS, 399, 539 
Calvelo, D. E., Fender, R. P., Russell, D. M., et al. 2010, MNRAS, 409, 839 Cantrell, A. G., Bailyn, C. D., Orosz, J. A., et al. 2010, ApJ, 710, 1127 Capitanio, F., Bazzano, A., Ubertini, P., et al. 2006, ApJ, 643, 376

Capitanio, F., Giroletti, M., Molina, M., et al. 2009, ApJ, 690, 1621

Casares, J. 1996, in IAU Colloq. 158: Cataclysmic Variables and Related Objects, eds. A. Evans, \& J. H. Wood, Astrophys. Space Sci. Lib., 208, 395

Casares, J., \& Charles, P. A. 1994, MNRAS, 271, L5

Casares, J., \& Jonker, P. G. 2014, Space Sci. Rev., 183, 223

Casares, J., Charles, P. A., Jones, D. H. P., Rutten, R. G. M., \& Callanan, P. J. 1991, MNRAS, 250, 712

Casares, J., Charles, P. A., \& Naylor, T. 1992, Nature, 355, 614

Casares, J., Charles, P. A., Naylor, T., \& Pavlenko, E. P. 1993, MNRAS, 265, 834

Casares, J., Charles, P. A., \& Marsh, T. R. 1995a, MNRAS, 277, L45

Casares, J., Martin, A. C., Charles, P. A., et al. 1995b, MNRAS, 276, L35

Casares, J., Martin, E. L., Charles, P. A., Molaro, P., \& Rebolo, R. 1997, New Astron., 1, 299

Casares, J., Zurita, C., Shahbaz, T., Charles, P. A., \& Fender, R. P. 2004, ApJ, 613, L133

Casares, J., Orosz, J. A., Zurita, C., et al. 2009, ApJS, 181, 238

Casares, J., Rodríguez-Gil, P., Zurita, C., et al. 2012, Atel, 4347, 1

Casares, J., Negueruela, I., Ribó, M., et al. 2014, Nature, 505, 378

Castro-Tirado, A. J., Brandt, S., \& Lund, N. 1992a, IAU Circ., 5590, 2

Castro-Tirado, A. J., Pavlenko, P., Shlyapnikov, A., et al. 1992b, IAU Circ., 5588,1

Castro-Tirado, A. J., Davies, J., Brandt, S., Lund, N., \& Everall, C. 1993, IAU Circ., 5830, 1

Castro-Tirado, A. J., Kilmartin, P., Gilmore, A., et al. 2001, IAU Circ., 7707, 3 Chakrabarty, D., Jonker, P. G., \& Markwardt, C. B. 2014, ATel, 6533, 1

Charles, P. A., \& Coe, M. J. 2006, Compact Stellar X-ray Sources, eds. M. Lewin, W. H. G., \& van der Klis (Cambridge University Press), 215

Charles, P. A., Kidger, M. R., Pavlenko, E. P., Prokof'eva, V. V., \& Callanan, P. J. 1991, MNRAS, 249, 567

Charles, P., Hassall, B., Machin, G., et al. 1988, IAU Circ., 4609, 1

Chaty, S., \& Bessolaz, N. 2006, A\&A, 455, 639

Chaty, S., Mirabel, I. F., Goldoni, P., et al. 2002, MNRAS, 331, 1065

Chaty, S., Mignani, R. P., \& Israel, G. L. 2006, MNRAS, 365, 1387

Chen, W., Shrader, C. R., \& Livio, M. 1997, ApJ, 491, 312

Chenevez, J., Brandt, S., Budtz-Jorgensen, C., et al. 2014a, ATel, 6530, 1

Chenevez, J., Brandt, S., Budtz-Jorgensen, C., et al. 2014b, ATel, 6602, 1

Chenevez, J., Vandbaek Kroer, L., Budtz-Jorgensen, C., et al. 2014c, ATel, 6451, 1

Chevalier, C., \& Ilovaisky, S. A. 1992, IAU Circ., 5520, 1

Chomiuk, L., Strader, J., Maccarone, T. J., et al. 2013, ApJ, 777, 69

Corbel, S., Kaaret, P., Jain, R. K., et al. 2001, ApJ, 554, 43

Corbel, S., Fender, R. P., Tomsick, J. A., Tzioumis, A. K., \& Tingay, S. 2004, ApJ, 617, 1272

Coriat, M., Broderick, J., Tzioumis, T., et al. 2012, ATel, 4067, 1

Corral-Santana, J. M., Casares, J., Shahbaz, T., et al. 2011, MNRAS, 413, L15

Corral-Santana, J. M., Casares, J., Muñoz-Darias, T., et al. 2013, Science, 339, 1048

Crowther, P. A., Barnard, R., Carpano, S., et al. 2010, MNRAS, 403, L41

Cummings, J. R., Barthelmy, S. D., Baumgartner, W. H., et al. 2012, GRB Coordinates Network, 13775, 1

Curran, P. A., Chaty, S., \& Zurita Heras, J. A. 2012, A\&A, 547, A41

D’Avanzo, P., Goldoni, P., Patruno, A., et al. 2010, ATel, 2900, 1

de Jong, J. A., van Paradijs, J., \& Augusteijn, T. 1996, A\&A, 314, 484

della Valle, M., \& Benetti, S. 1993, IAU Circ., 5890, 2

della Valle, M., Jarvis, B. J., \& West, R. M. 1991, A\&A, 247, L33

della Valle, M., Mirabel, I. F., \& Rodriguez, L. F. 1994, A\&A, 290, 803

della Valle, M., Benetti, S., Cappellaro, E., \& Wheeler, C. 1997, A\&A, 318, 179

Dhawan, V., Mirabel, I. F., \& Rodríguez, L. F. 2000, ApJ, 543, 373

Dubath, P., Revnivtsev, M., Goldoni, P., et al. 2003, IAU Circ., 8100, 1

Duerbeck, H. W. 1984, Ap\&SS, 99, 363

Dunn, R. J. H., Fender, R. P., Körding, E. G., Belloni, T., \& Cabanac, C. 2010, MNRAS, 403, 61

Dwek, E., Arendt, R. G., Hauser, M. G., et al. 1995, ApJ, 445, 716

Eachus, L. J., Wright, E. L., \& Liller, W. 1976, ApJ, 203, L17

Eikenberry, S. S., \& Fazio, G. G. 1995, IAU Circ., 6267, 2

Eikenberry, S. S., Cameron, P. B., Fierce, B. W., et al. 2001, ApJ, 561, 1027

Elvis, M., Griffiths, C. G., Turner, M. J. L., \& Page, C. G. 1975, IAU Circ., 2814, 1

Fabrika, S. 2004, Astrophys. Space Phys. Rev., 12,

Farr, W. M., Sravan, N., Cantrell, A., et al. 2011, ApJ, 741, 103

Fender, R. P., Garrington, S. T., McKay, D. J., et al. 1999, MNRAS, 304, 865

Fender, R. P., Hjellming, R. M., Tilanus, R. P. J., et al. 2001, MNRAS, 322, L23

Fender, R., Garrington, S., \& Muxlow, T. 2005, ATel, 558, 1
Filippenko, A. V., Matheson, T., Leonard, D. C., Barth, A. J., \& van Dyk, S. D. 1997, PASP, 109, 461

Filippenko, A. V., Leonard, D. C., Matheson, T., et al. 1999, PASP, 111, 969

Filippova, E., Bozzo, E., \& Ferrigno, C. 2014, A\&A, 563, A124

Francey, R. J. 1971, Nature Physical Science, 229, 229

Frank, J., King, A., \& Raine, D. J. 2002, Accretion Power in Astrophysics: Third Edition (Cambridge University Press)

Frei, Z., \& Gunn, J. E. 1994, AJ, 108, 1476

Froning, C. S., \& Robinson, E. L. 2001, AJ, 121, 2212

Fryer, C. L., \& Kalogera, V. 2001, ApJ, 554, 548

Fryer, C. L., Belczynski, K., Wiktorowicz, G., et al. 2012, ApJ, 749, 91

Gallo, E., Corbel, S., Fender, R. P., Maccarone, T. J., \& Tzioumis, A. K. 2004 MNRAS, 347, L52

Gallo, E., Fender, R. P., Miller-Jones, J. C. A., et al. 2006, MNRAS, 370, 1351

Garcia, M. R., \& Wilkes, B. J. 2002, ATel, 104, 1

Garcia, M. R., Callanan, P. J., McClintock, J. E., \& Zhao, P. 1996, ApJ, 460, 932

Garcia, M., Brown, W., Pahre, M., et al. 2000, IAU Circ., 7392, 2

Garnavich, P. M., Stanek, K. Z., \& Berlind, P. 1999, IAU Circ., 7276, 1

Gelino, D. M. 2002, in BAAS, 34, Am. Astron. Soc. Meet. Abstr., 200, 654

Gelino, D. M., \& Harrison, T. E. 2003, ApJ, 599, 1254

Gelino, D. M., Harrison, T. E., \& McNamara, B. J. 2001a, AJ, 122, 971

Gelino, D. M., Harrison, T. E., \& Orosz, J. A. 2001b, AJ, 122, 2668

Gelino, D. M., Balman, Ş., Kızıloğlu, Ü., et al. 2006, ApJ, 642, 438

Gelino, D. M., Gelino, C. R., \& Harrison, T. E. 2010, ApJ, 718, 1

Giacconi, R., Gursky, H., Paolini, F. R., \& Rossi, B. B. 1962, Phys. Rev. Lett., 9, 439

Giacconi, R., Gorenstein, P., Gursky, H., \& Waters, J. R. 1967, ApJ, 148, L119

Giacconi, R., Murray, S., Gursky, H., et al. 1972, ApJ, 178, 281

Giacconi, R., Murray, S., Gursky, H., et al. 1974, ApJS, 27, 37

Goldwurm, A., Paul, J., Mandrou, P., et al. 1992, IAU Circ., 5589, 1

González Hernández, J. I., \& Casares, J. 2010, A\&A, 516, A58

González Hernández, J. I., Rebolo, R., \& Israelian, G. 2008a, A\&A, 478, 203

González Hernández, J. I., Rebolo, R., Israelian, G., et al. 2008b, ApJ, 679, 732

Gottwald, M., Steinle, H., Pietsch, W., \& Graser, U. 1991, A\&AS, 89, 367

Grebenev, S. A., Molkov, S. V., \& Sunyaev, R. A. 2005, ATel, 444, 1

Grebenev, S. A., Molkov, S. V., Revnivtsev, M. G., \& Sunyaev, R. A. 2007, ESA SP, 622, 373

Greene, J., Bailyn, C. D., \& Orosz, J. A. 2001, ApJ, 554, 1290

Greiner, J., Dennerl, K., \& Predehl, P. 1996, A\&A, 314, L21

Greiner, J., Rau, A., \& Arne, S. 2012, ATel, 4030, 1

Griffiths, R. E., Bradt, H., Doxsey, R., et al. 1978, ApJ, 221, L63

Hakala, P., Muhli, P., \& Charles, P. 2014, MNRAS, 444, 3802

Halpern, J. P. 2005, ATel, 549, 1

Harlaftis, E. T., \& Greiner, J. 2004, A\&A, 414, L13

Harlaftis, E. T., Horne, K., \& Filippenko, A. V. 1996, PASP, 108, 762

Harlaftis, E. T., Steeghs, D., Horne, K., \& Filippenko, A. V. 1997, AJ, 114, 1170

Harlaftis, E., Collier, S., Horne, K., \& Filippenko, A. V. 1999, A\&A, 341, 491

Harmon, B. A., Wilson, R. B., Finger, M. H., et al. 1992, IAU Circ., 5504, 1

Harmon, B. A., Zhang, S. N., Fishman, G. J., \& Paciesas, W. S. 1993a, IAU Circ., 5864,1

Harmon, B. A., Zhang, S. N., Paciesas, W. S., \& Fishman, G. J. 1993b, IAU Circ., 5874,1

Harries, J. R., McCracken, K. G., Francey, R. J., \& Fenton, A. G. 1967, Nature, 215, 38

Heinke, C. O., Bahramian, A., Altamirano, D., \& Wijnands, R. 2014, ATel, 6459, 1

Hjellming, R. M., \& Rupen, M. P. 1995, Nature, 375, 464

Hjellming, R. M., Calovini, T. A., Han, X. H., \& Cordova, F. A. 1988, ApJ, 335 , L75

Hjellming, R. M., Rupen, M. P., Marti, J., Mirabel, F., \& Rodriguez, L. F. 1996, IAU Circ., 6383, 1

Hjellming, R. M., Rupen, M. P., \& Mioduszewski, A. J. 1998a, IAU Circ., 6934, 2

Hjellming, R. M., Rupen, M. P., Mioduszewski, A. J., et al. 1998b, in BAAS, 30, 103.08

Holt, S. S., Kaluzienski, L. J., Boldt, E. A., \& Serlemitsos, P. J. 1974, IAU Circ., 2729,1

Homan, J., \& Wijnands, R. 2003, ATel, 169, 1

Homan, J., Buxton, M., Markoff, S., et al. 2005, ApJ, 624, 295

Homan, J., Wijnands, R., Kong, A., et al. 2006, MNRAS, 366, 235

Horne, K., Harlaftis, E. T., Baptista, R., et al. 1996, IAU Circ., 6406, 2

Hynes, R. I. 2005, ApJ, 623, 1026

Hynes, R. I., Roche, P., Charles, P. A., \& Coe, M. J. 1999, MNRAS, 305, L49

Hynes, R. I., Haswell, C. A., Chaty, S., Shrader, C. R., \& Cui, W. 2002, MNRAS, 331,169

Hynes, R. I., Charles, P. A., Casares, J., et al. 2003a, MNRAS, 340, 447 
Hynes, R. I., Steeghs, D., Casares, J., Charles, P. A., \& O’Brien, K. 2003b, ApJ, 583, L95

Hynes, R. I., Steeghs, D., Casares, J., Charles, P. A., \& O’Brien, K. 2004, ApJ, 609,317

Hynes, R. I., Britt, C. T., Jonker, P. G., Wijnands, R., \& Greiss, S. 2012, ATel, 4417,1

in 't Zand, J., Heise, J., Bazzano, A., et al. 1999, IAU Circ., 7119, 1

in 't Zand, J. J. M., Kaptein, R. G., \& Heise, J. 2001, IAU Circ., 7582, 1

in 't Zand, J. J. M., Markwardt, C. B., Bazzano, A., et al. 2002a, A\&A, 390, 597

in't Zand, J. J. M., Miller, J. M., Oosterbroek, T., \& Parmar, A. N. 2002b, A\&A, 394,553

in’t Zand, J. J. M., Heise, J., Lowes, P., \& Ubertini, P. 2003, ATel, 160, 1

Ioannou, Z., Robinson, E. L., Welsh, W. F., \& Haswell, C. A. 2004, AJ, 127, 481

Jain, R. K., Bailyn, C. D., Orosz, J. A., McClintock, J. E., \& Remillard, R. A. 2001a, ApJ, 554, L181

Jain, R. K., Bailyn, C. D., Orosz, J. A., et al. 2001b, ApJ, 546, 1086

Jaisawal, G. K., Homan, J., Naik, S., \& Jonker, P. 2015, ATel, 7361, 1

Jonker, P. G., \& Nelemans, G. 2004, MNRAS, 354, 355

Kalberla, P. M. W., Burton, W. B., Hartmann, D., et al. 2005, A\&A, 440, 775

Kaluzienski, L. J., \& Holt, S. S. 1977, IAU Circ., 3104, 3

Kaluzienski, L. J., Holt, S. S., Boldt, E. A., et al. 1975, ApJ, 201, L121

Kawashima, T., Ohsuga, K., Mineshige, S., et al. 2012, ApJ, 752, 18

Kennea, J. A., \& Capitanio, F. 2007, ATel, 1140, 1

Kennea, J. A., Wijnands, R., Burrows, D. N., Nousek, J., \& Gehrels, N. 2006a, ATel, 921, 1

Kennea, J. A., Wijnands, R., Burrows, D. N., Nousek, J., \& Gehrels, N. 2006b, ATel, 920, 1

Kennea, J. A., Esposito, P., Israel, G. L., et al. 2011a, ATel, 3336, 1

Kennea, J. A., Evans, P. A., Krimm, H. A., et al. 2011b, ATel, 3331,

Kennea, J. A., Linares, M., Krimm, H. A., et al. 2013, ATel, 5479, 1

Khargharia, J., Froning, C. S., \& Robinson, E. L. 2010, ApJ, 716, 1105

Khargharia, J., Froning, C. S., Robinson, E. L., \& Gelino, D. M. 2013, AJ, 145, 21

Kiel, P. D., \& Hurley, J. R. 2006, MNRAS, 369, 1152

King, A. R., \& Wijnands, R. 2006, MNRAS, 366, L31

King, A. R., Kolb, U., \& Burderi, L. 1996a, ApJ, 464, L127

King, N. L., Harrison, T. E., \& McNamara, B. J. 1996b, AJ, 111, 1675

Kitamoto, S., Miyamoto, S., Tsunemi, H., Makishima, K., \& Nakagawa, M. 1984, PASJ, 36, 799

Kitamoto, S., Tsunemi, H., Pedersen, H., Ilovaisky, S. A., \& van der Klis, M 1990, ApJ, 361, 590

Kochanek, C. S. 2014, ApJ, 785, 28

Kong, A. K. H. 2012, ApJ, 760, L27

Kreidberg, L., Bailyn, C. D., Farr, W. M., \& Kalogera, V. 2012, ApJ, 757, 36

Krimm, H. A., Barthelmy, S. D., Barbier, L., et al. 2007, ATel, 1093, 1

Krimm, H. A., Barthelmy, S. D., Baumgartner, W., et al. 2008a, ATel, 1855, 1

Krimm, H. A., Kennea, J., Barthelmy, S. D., et al. 2008b, ATel, 1610, 1

Krimm, H. A., Racusin, J. L., \& Markwardt, C. B. 2008c, ATel, 1706, 1

Krimm, H. A., Kennea, J. A., Schady, P., \& Evans, P. A. 2009, ATel, 1893, 1

Krimm, H. A., Barthelmy, S. D., Baumgartner, W., et al. 2011a, ATel, 3144, 1

Krimm, H. A., Barthelmy, S. D., Baumgartner, W., et al. 2011b, ATel, 3138, 1

Krimm, H. A., Tomsick, J. A., Markwardt, C. B., et al. 2011c, ApJ, 735, 104

Krimm, H. A., Barthelmy, S. D., Baumgartner, W., et al. 2012, ATel, 4139, 1

Krimm, H. A., Barthelmy, S. D., Baumgartner, W., et al. 2013, ATel, 4769, 1

Kuulkers, E., Parmar, A. N., Kitamoto, S., Cominsky, L. R., \& Sood, R. K. 1997, MNRAS, 291, 81

Kuulkers, E., Lutovinov, A., Parmar, A., et al. 2003, ATel, 149, 1

Kuulkers, E., Kouveliotou, C., Belloni, T., et al. 2013, A\&A, 552, A32

Lapshov, I., Sazonov, S., \& Sunyaev, R. 1993, IAU Circ., 5864, 1

Lattimer, J. M., \& Prakash, M. 2005, Phys. Rev. Lett., 94, 111101

Laycock, S. G. T., Maccarone, T. J., \& Christodoulou, D. M. 2015, MNRAS, 452, L31

Levine, A. M., \& Remillard, R. A. 2007, ATel, 1024, 1

Levine, A. M., Lin, D., \& Remillard, R. A. 2005a, ATel, 623, 1

Levine, A. M., Swank, J. H., Lin, D., \& Remillard, R. A. 2005b, ATel, 578, 1

Lewis, F., Russell, D. M., \& Shahbaz, T. 2012, ATel, 4162, 1

Liu, Q. Z., van Paradijs, J., \& van den Heuvel, E. P. J. 2006, A\&A, 455, 1165

Liu, Q. Z., van Paradijs, J., \& van den Heuvel, E. P. J. 2007, A\&A, 469, 807

Longmore, A., Cannon, R., Murdin, P., et al. 1977, IAU Circ., 3110, 2

Lund, N., Brandt, S., Makino, F., et al. 1991, IAU Circ., 5161, 1

MacDonald, R. K. D., Bailyn, C. D., Buxton, M., et al. 2014, ApJ, 784, 2

Macias, P., Orosz, J. A., Bailyn, C. D., et al. 2011, in BAAS, 43, 143.04

Maitra, D., Cobb, B., Bailyn, C., Nelan, J., \& Gonzalez, D. 2005, ATel, 628, 1

Makino, F. 1987, IAU Circ., 4342, 1

Makino, F. 1988, IAU Circ., 4571, 2

Makino, F. 1989, IAU Circ., 4782, 1

Makino, F., \& GINGA, T. 1988, IAU Circ., 4587, 1
Makino, F., McNaught, R. H., Jones, A., \& West, R. M. 1991, IAU Circ., 5161, 1

Mandrou, P. 1990, IAU Circ., 5032, 1

Markert, T. H., Canizares, C. R., Clark, G. W., et al. 1973, ApJ, 184, L67

Markwardt, C. B. 2003, ATel, 143, 1

Markwardt, C. B. 2009, in Astronomical Data Analysis Software and Systems XVIII, eds. D. A. Bohlender, D. Durand, \& P. Dowler, ASP Conf. Ser., 411, 251

Markwardt, C. B., \& Swank, J. H. 2003, ATel, 133, 1

Markwardt, C. B., \& Swank, J. H. 2007, ATel, 1235, 1

Markwardt, C. B., Marshall, F. E., \& Swank, J. 1999, IAU Circ., 7274, 1

Markwardt, C. B., Strohmayer, T. E., Swank, J. H., \& Levine, A. M. 2005, ATel, 579,1

Markwardt, C. B., Halpern, J. P., Holland, S. T., et al. 2008, ATel, 1716, 1

Markwardt, C. B., Beardmore, A. P., Miller, J., \& Swank, J. H. 2009a, ATel, 2120,1

Markwardt, C. B., Swank, J. H., Barthelmy, S. D., et al. 2009b, ATel, 2258, 1

Markwardt, C. B., Swank, J. H., Krimm, H. A., Pereira, D., \& Strohmayer, T. E. 2009c, ATel, 2107, 1

Marshall, F. E., \& Smith, D. 1997, IAU Circ., 6603, 1

Marshall, F. E., Ebisawa, K., Remillard, R., \& Valinia, A. 1996, IAU Circ., 6504, 2

Marti, J., Mirabel, I. F., Duc, P.-A., \& Rodriguez, L. F. 1997, A\&A, 323, 158

Martin, A. C., Casares, J., Charles, P. A., van der Hooft, F., \& van Paradijs, J. 1995, MNRAS, 274, L46

Mason, K. O., Parmar, A. N., \& White, N. E. 1985, MNRAS, 216, 1033

Mata Sánchez, D., Muñoz-Darias, T., Casares, J., Corral-Santana, J. M., \& Shahbaz, T. 2015, MNRAS, 454, 2199

Matilsky, T. A., Giacconi, R., Gursky, H., Kellogg, E. M., \& Tananbaum, H. D. 1972, ApJ, 174, L53

Matsuoka, M., Kawasaki, K., Ueno, S., et al. 2009, PASJ, 61, 999

McClintock, J. E., \& Remillard, R. A. 2006, Compact stellar X-ray sources, eds. W. H. G. Lewin, \& M. van der Klis (Cambridge Astrophysical Series), 39, 157

McClintock, J. E., Shafee, R., Narayan, R., et al. 2006, ApJ, 652, 518

McClintock, J. E., Remillard, R. A., Rupen, M. P., et al. 2009, ApJ, 698, 1398

Mellinger, A. 2009, PASP, 121, 1180

Menou, K., Hameury, J.-M., \& Stehle, R. 1999, MNRAS, 305, 79

Mereghetti, S., Cremonesi, D. I., Haardt, F., et al. 1997, ApJ, 476, 829

Miller, J. M., \& Remillard, R. A. 2002, ATel, 98, 1

Miller, J. M., Fabbiano, G., Miller, M. C., \& Fabian, A. C. 2003, ApJ, 585, L37

Miller, J. M., Reynolds, M. T., \& Kennea, J. 2015a, ATel, 7612, 1

Miller, J. M., Tomsick, J. A., Bachetti, M., et al. 2015b, ApJ, 799, L6

Miller-Jones, J. C. A., \& Sivakoff, G. R. 2012, ATel, 4394, 1

Miller-Jones, J. C. A., Jonker, P. G., Dhawan, V., et al. 2009, ApJ, 706, L230

Miller-Jones, J. C. A., Jonker, P. G., Ratti, E. M., et al. 2011a, MNRAS, 415, 306

Miller-Jones, J. C. A., Tzioumis, A. K., Jonker, P. G., et al. 2011b, ATel, 3364, 1

Miller-Jones, J. C. A., Sivakoff, G. R., Altamirano, D., et al. 2012, MNRAS, 421, 468

Miller-Jones, J. C. A., Sivakoff, G. R., \& Krimm, H. A. 2013, ATel, 5530, 1

Mirabel, I. F., Cordier, B., Bonibaker, J., Stirpe, G., \& Rodriguez, L. F. 1993, IAU Circ., 5876, 1

Morihana, K., Sugizaki, M., Nakahira, S., et al. 2013, PASJ, 65, L10

Morris, D. C., Burrows, D. N., Racusin, J., et al. 2005, ATel, 552, 1

Muñoz-Darias, T., Casares, J., \& Martínez-Pais, I. G. 2008, MNRAS, 385, 2205

Muñoz-Darias, T., Motta, S., Stiele, H., \& Belloni, T. M. 2011, MNRAS, 415, 292

Muñoz-Darias, T., de Ugarte Postigo, A., Russell, D. M., et al. 2013, MNRAS, 432, 1133

Muñoz-Darias, T., Fender, R. P., Motta, S. E., \& Belloni, T. M. 2014, MNRAS, 443, 3270

Mukai, K. 1993, Legacy, 3, 21

Muno, M. P., Pfahl, E., Baganoff, F. K., et al. 2005, ApJ, 622, L113

Murdin, P., Griffiths, R. E., Pounds, K. A., Watson, M. G., \& Longmore, A. J. 1977, MNRAS, 178, 27

Nagata, T., Kato, D., Baba, D., et al. 2003, PASJ, 55, L73

Nakahira, S., Tomida, H., Negoro, H., et al. 2013, ATel, 5474, 1

Narayan, R., \& McClintock, J. E. 2005, ApJ, 623, 1017

Negoro, H., Yamaoka, K., Nakahira, S., et al. 2010, ATel, 2873, 1

Negoro, H., Nakahira, S., Ueda, Y., et al. 2011a, ATel, 3330, 1

Negoro, H., Nakajima, M., Nakahira, S., et al. 2011b, ATel, 3611, 1

Negoro, H., Itoh, D., Ueno, S., et al. 2015, ATel, 7579, 1

Neustroev, V. V., Veledina, A., Poutanen, J., et al. 2014, MNRAS, 445, 2424

Nowak, M. A., Juett, A., Homan, J., et al. 2008, ApJ, 689, 1199

Nowak, M. A., Wilms, J., Pottschmidt, K., et al. 2012, ApJ, 744, 107

O'Brien, K., Clarke, F., Fender, R., et al. 2003, ATel, 117, 1 
Okamura, S., \& Noguchi, T. 1988, IAU Circ., 4589, 1

Orosz, J. A. 2003, in A Massive Star Odyssey: From Main Sequence to Supernova, eds. K. van der Hucht, A. Herrero, \& C. Esteban, IAU Symp., 212,365

Orosz, J. A., \& Bailyn, C. D. 1997, ApJ, 477, 876

Orosz, J. A., Bailyn, C. D., Remillard, R. A., McClintock, J. E., \& Foltz, C. B. 1994, ApJ, 436, 848

Orosz, J. A., Bailyn, C. D., McClintock, J. E., \& Remillard, R. A. 1996, ApJ, 468,380

Orosz, J. A., Jain, R. K., Bailyn, C. D., McClintock, J. E., \& Remillard, R. A. 1998, ApJ, 499, 375

Orosz, J. A., Kuulkers, E., van der Klis, M., et al. 2001, ApJ, 555, 489

Orosz, J. A., Groot, P. J., van der Klis, M., et al. 2002, ApJ, 568, 845

Orosz, J. A., McClintock, J. E., Remillard, R. A., \& Corbel, S. 2004, ApJ, 616, 376

Orosz, J. A., McClintock, J. E., Narayan, R., et al. 2007, Nature, 449, 872

Orosz, J. A., Steeghs, D., McClintock, J. E., et al. 2009, ApJ, 697, 573

Orosz, J. A., McClintock, J. E., Aufdenberg, J. P., et al. 2011a, ApJ, 742, 84

Orosz, J. A., Steiner, J. F., McClintock, J. E., et al. 2011b, ApJ, 730, 75

Orosz, J. A., Steiner, J. F., McClintock, J. E., et al. 2014, ApJ, 794, 154

Ozawa, H., Suwa, F., Negoro, H., et al. 2011, ATel, 3098, 1

Özel, F., Psaltis, D., Narayan, R., \& McClintock, J. E. 2010, ApJ, 725, 1918

Özel, F., Psaltis, D., Narayan, R., \& Santos Villarreal, A. 2012, ApJ, 757, 55

Paciesas, W. S., Briggs, M. S., Harmon, B. A., Wilson, R. B., \& Finger, M. H. 1992, IAU Circ., 5580, 1

Paizis, A., Nowak, M. A., Chaty, S., et al. 2007, ApJ, 657, L109

Paizis, A., Nowak, M. A., Rodriguez, J., et al. 2015, ApJ, 808, 34

Palmer, D. M., Barthelmey, S. D., Cummings, J. R., et al. 2005, ATel, 546, 1

Paragi, Z., van der Horst, A. J., Granot, J., et al. 2010, ATel, 2906, 1

Park, S. Q., Miller, J. M., McClintock, J. E., et al. 2004, ApJ, 610, 378

Parmar, A. N., \& White, N. E. 1985, IAU Circ., 4051, 1

Parmar, A. N., Angelini, L., Roche, P., \& White, N. E. 1993, A\&A, 279, 179

Paul, J., Bouchet, L., Churazov, E., \& Sunyaev, R. 1996, IAU Circ., 6348, 1

Pedersen, H., Veron, M., Veron, P., \& Schuster, H.-E. 1983, IAU Circ., 3858, 1

Podsiadlowski, P., Rappaport, S., \& Pfahl, E. D. 2002, ApJ, 565, 1107

Pounds, K. A., Holt, S. S., Kaluzienski, L. J., Boldt, E. A., \& Serlemitsos, P. J.

1974, IAU Circ., 2729, 1

Poutanen, J., Lipunova, G., Fabrika, S., Butkevich, A. G., \& Abolmasov, P. 2007, MNRAS, 377, 1187

Priedhorsky, W. 1986, Ap\&SS, 126, 89

Pylyser, E., \& Savonije, G. J. 1988, A\&A, 191, 57

Rahoui, F., Tomsick, J. A., Coriat, M., et al. 2015, ApJ, 810, 161

Ratti, E. M., Jonker, P. G., Miller-Jones, J. C. A., et al. 2012, MNRAS, 423, 2656

Rau, A., Greiner, J., Elliott, J., \& Olivares E, F. 2011a, ATel, 3365, 1

Rau, A., Greiner, J., \& Filgas, R. 2011b, ATel, 3140, 1

Rau, A., Greiner, J., \& Schady, P. 2012, ATel, 4144, 1

Rau, A., Knust, F., Schmidl, S., \& Greiner, J. 2013a, ATel, 4904, 1

Rau, A., Tanga, M., \& Greiner, J. 2013b, ATel, 5482, 1

Reid, M. J., McClintock, J. E., Steiner, J. F., et al. 2014, ApJ, 796, 2

Reis, R. C., Reynolds, M. T., Miller, J. M., et al. 2013, ApJ, 778, 155

Remillard, R. 2001, IAU Circ., 7707, 1

Remillard, R. A., \& McClintock, J. E. 2006, ARA\&A, 44, 49

Remillard, R. A., Orosz, J. A., McClintock, J. E., \& Bailyn, C. D. 1996, ApJ, 459, 226

Remillard, R., Levine, A., Swank, J., \& Strohmayer, T. 1997a, IAU Circ., 6710,

Remillard, R., Marshall, F., \& Takeshima, T. 1997b, IAU Circ., 6772, 1

Remillard, R., Levine, A., Wood, A., et al. 1998, IAU Circ., 6920, 1

Remillard, R., Morgan, E., Smith, D., \& Smith, E. 2000, IAU Circ., 7389, 2

Remillard, R. A., Levine, A. M., Morgan, E. H., Smith, E., \& Swank, J. 2003, ATel, 114, 1

Remillard, R., Levine, A. M., Morgan, E. H., Markwardt, C. B., \& Swank, J. H. 2006, ATel, 714, 1

Revnivtsev, M., Gilfanov, M., \& Churazov, E. 1998a, A\&A, 339, 483

Revnivtsev, M., Gilfanov, M., Churazov, E., et al. 1998b, A\&A, 331, 557

Revnivtsev, M. G., Trudolyubov, S. P., \& Borozdin, K. N. 2000, MNRAS, 312, 151

Revnivtsev, M., Gilfanov, M., Churazov, E., \& Sunyaev, R. 2003, ATel, 150, 1

Richter, G. A. 1989, IBVS, 3362, 1

Ritter, H., \& King, A. R. 2002, in The Physics of Cataclysmic Variables and Related Objects, eds. B. T. Gänsicke, K. Beuermann, \& K. Reinsch, ASP Conf. Ser., 261, 531

Ritter, H., \& Kolb, U. 2003, A\&A, 404, 301

Roberts, M. S. E., Michelson, P. F., Cominsky, L. R., et al. 1996, IAU Circ., 6302, 2

Robertson, B. S. C., Warren, P. R., \& Bywater, R. A. 1976, IBVS, 1173, 1

Rodriguez, J., Corbel, S., Caballero, I., et al. 2011, A\&A, 533, L4

Romani, R. W. 1998, A\&A, 333, 583
Rupen, M. P., Dhawan, V., \& Mioduszewski, A. J. 2005a, ATel, 589, 1 Rupen, M. P., Mioduszewski, A. J., \& Dhawan, V. 2005b, ATel, 490, 1 Rupen, M. P., Dhawan, V., \& Mioduszewski, A. J. 2006, ATel, 721, 1 Russell, D. M., \& Lewis, F. 2015, ATel, 7637, 1

Russell, D. M., Lewis, F., Bersier, D., et al. 2010, ATel, 2884, 1

Russell, D. M., Russell, T. D., Miller-Jones, J. C. A., et al. 2013, ApJ, 768, L35 Russell, T. D., Soria, R., Motch, C., et al. 2014, MNRAS, 439, 1381

Russell, T. D., Miller-Jones, J. C. A., Curran, P. A., et al. 2015, MNRAS, 450, 1745

Sala, G., Greiner, J., Ajello, M., Bottacini, E., \& Haberl, F. 2007, A\&A, 473, 561

Sala, G., Greiner, J., Ajello, M., \& Primak, N. 2008, A\&A, 489, 1239

Samus, N. N., Hazen, M., Williams, D., et al. 1999, IAU Circ., 7277, 1

Sato, R., Serino, M., Nakahira, S., et al. 2012, ATel, 4024, 1

Schlafly, E. F., \& Finkbeiner, D. P. 2011, ApJ, 737, 103

Schlegel, D. J., Finkbeiner, D. P., \& Davis, M. 1998, ApJ, 500, 525

Shahbaz, T., \& Kuulkers, E. 1998, MNRAS, 295, L1

Shahbaz, T., Ringwald, F. A., Bunn, J. C., et al. 1994, MNRAS, 271, L10

Shahbaz, T., van der Hooft, F., Charles, P. A., Casares, J., \& van Paradijs, J. 1996, MNRAS, 282, L47

Shahbaz, T., Naylor, T., \& Charles, P. A. 1997, MNRAS, 285, 607

Shahbaz, T., van der Hooft, F., Casares, J., Charles, P. A., \& van Paradijs, J. 1999, MNRAS, 306, 89

Shahbaz, T., Fender, R., \& Charles, P. A. 2001, A\&A, 376, L17

Shahbaz, T., Zurita, C., Casares, J., et al. 2003, ApJ, 585, 443

Shahbaz, T., Dhillon, V. S., Marsh, T. R., et al. 2005, MNRAS, 362, 975

Shahbaz, T., Fender, R. P., Watson, C. A., \& O’Brien, K. 2008, ApJ, 672, 510

Shahbaz, T., Dhillon, V. S., Marsh, T. R., et al. 2010, MNRAS, 403, 2167

Shahbaz, T., Russell, D. M., Zurita, C., et al. 2013, MNRAS, 434, 2696

Shaposhnikov, N., \& Titarchuk, L. 2009, ApJ, 699, 453

Shidatsu, M., Ueda, Y., Nakahira, S., et al. 2013, ApJ, 779, 26

Shrader, C. R., Wagner, R. M., Hjellming, R. M., Han, X. H., \& Starrfield, S. G. 1994, ApJ, 434, 698

Silverman, J. M., \& Filippenko, A. V. 2008, ApJ, 678, L17

Skinner, G. K., Foster, A. J., Willmore, A. P., \& Eyles, C. J. 1990, MNRAS, 243 72

Smith, D. A. 1998, IAU Circ., 7008, 1

Smith, D. A., Levine, A., \& Wood, A. 1998, ATel, 25, 1

Smith, D. A., Levine, A. M., Remillard, R., et al. 2000, IAU Circ., 7399, 1

Soldi, S., Walter, R., Eckert, D., et al. 2006, ATel, 885, 1

Soleri, P., Muñoz-Darias, T., Motta, S., et al. 2013, MNRAS, 429, 1244

Steeghs, D., Miller, J. M., Kaplan, D., \& Rupen, M. 2003, ATel, 146, 1

Steeghs, D., Torres, M. A. P., Jonker, P. G., et al. 2005a, ATel, 494, 1

Steeghs, D., Torres, M. A. P., Jonker, P. G., et al. 2005b, ATel, 478, 1

Steeghs, D., Torres, M. A. P., Koviak, K., McCarthy, P., \& Jonker, P. G. 2005c, ATel, 629, 1

Steeghs, D., Torres, M. A. P., Pych, W., \& Thompson, I. 2005d, ATel, 585,

Steeghs, D., McClintock, J. E., Parsons, S. G., et al. 2013, ApJ, 768, 185

Stephenson, C. B., \& Sanduleak, N. 1977, ApJS, 33, 459

Stiele, H., Munoz-Darias, T., Motta, S., \& Belloni, T. 2011, ArXiv e-prints [arXiv: 1103.4312]

Stiele, H., Muñoz-Darias, T., Motta, S., \& Belloni, T. M. 2012, MNRAS, 422, 679

Strader, J., Chomiuk, L., Maccarone, T. J., Miller-Jones, J. C. A., \& Seth, A. C. 2012, Nature, 490, 71

Strohmayer, T., \& Marshall, F. E. 1998, IAU Circ., 6934, 2

Sunyaev, R., Churazov, E., Gilfanov, M., et al. 1991, ApJ, 383, L49

Sunyaev, R., Churazov, E., Gilfanov, M., et al. 1992, ApJ, 389, L75

Sunyaev, R., Churazov, E., Revnivtsev, M., et al. 1997, IAU Circ., 6599, 2

Suzuki, K., Fukushima, K., Negoro, H., et al. 2015, ATel, 7233, 1

Tanaka, Y. 1992, Institute of Space and Astronautical Science (ISAS), 19

Tanaka, Y., \& Shibazaki, N. 1996, ARA\&A, 34, 607

Tomsick, J. A., Smith, E., Swank, J., Wijnands, R., \& Homan, J. 2001, IAU Circ., 7575,2

Tomsick, J. A., Kalemci, E., \& Kaaret, P. 2004, ApJ, 601, 439

Tomsick, J. A., Corbel, S., Goldwurm, A., \& Kaaret, P. 2005, ApJ, 630, 413

Tomsick, J. A., DelSanto, M., \& Belloni, T. 2012, ATel, 4393, 1

Torres, M. A. P., Callanan, P. J., et al. 2002, ApJ, 569, 423

Torres, M. A. P., Callanan, P. J., Garcia, M. R., et al. 2004, ApJ, 612, 1026

Torres, M. A. P., Steeghs, D., Jonker, P. G., Burns, C. R., \& Freedman, W. L. 2006a, ATel, 909, 1

Torres, M. A. P., Steeghs, D., Jonker, P. G., et al. 2006b, ATel, 733, 1

Torres, M. A. P., Steeghs, D., Jonker, P. G., et al. 2007a, ATel, 1252, 1

Torres, M. A. P., Steeghs, D., Jonker, P. G., et al. 2007b, ATel, 1072, 1

Torres, M. A. P., Steeghs, D., Jonker, P. G., Greene, J. E., \& Hainline, K. N. 2008, ATel, 1720,

Torres, M. A. P., Steeghs, D., Jonker, P. G., et al. 2009a, ATel, 2190, 1

Torres, M. A. P., Steeghs, D., Jonker, P. G., Thompson, I., \& Soderberg, A. M. 2009b, ATel, 2268, 1 
Torres, M. A. P., Jonker, P. G., Steeghs, D., \& Mulchaey, J. S. 2011, ATel, 3150, 1

Torres, M. A. P., Jonker, P. G., Miller-Jones, J. C. A., et al. 2015, MNRAS, 450, 4292

Tsunemi, H., Kitamoto, S., Okamura, S., \& Roussel-Dupre, D. 1989, ApJ, 337, L81

Turler, M., Cadolle Bel, M., Diehl, R., et al. 2005, ATel, 624, 1

Ueda, Y., Dotani, T., Uno, S., et al. 1997, IAU Circ., 6627, 2

Usui, R., Nakahira, S., Tomida, H., et al. 2012, ATel, 4140, 1

Val-Baker, A. K. F., Norton, A. J., \& Negueruela, I. 2007, in The Multicolored Landscape of Compact Objects and Their Explosive Origins, eds. T. di Salvo, G. L. Israel, L. Piersant, et al., AIP Conf. Ser., 924, 530

van den Heuvel, E. P. J. 1992, in X-ray Binaries and Recycled Pulsars (Kluwer Academic Publishers), 233

van den Heuvel, E. P. J. 2001, in Evolution of Binary and Multiple Star Systems, eds. P. Podsiadlowski, S. Rappaport, A. R. King, F. D'Antona, \& L. Burderi, ASP Conf. Ser., 229, 525

van der Hooft, F., Heemskerk, M. H. M., Alberts, F., \& van Paradijs, J. 1998, A\&A, 329, 538

Vargas, M., Goldwurm, A., Paul, J., et al. 1996, A\&A, 313, 828

Voges, W., Aschenbach, B., Boller, T., et al. 1999, A\&A, 349, 389

Wachmann, A. A. 1948 (Berlin: Akademie-Verlag)

Wachter, S., \& Smale, A. P. 1998, ApJ, 496, L21

Wagner, R. M., \& Starrfield, S. 2002, ATel, 86, 1

Wagner, R. M., Henden, A. A., Bertram, R., \& Starrfield, S. G. 1988, IAU Circ., 4600, 2

Wagner, R. M., Bertram, R., Starrfield, S. G., \& Shrader, C. R. 1992, IAU Circ., 5589,1
Wang, X., \& Wang, Z. 2014, ApJ, 788, 184

Wang, Z., Baganoff, F. K., Muno, M., et al. 2006, ATel, 935, 1

Warwick, R. S., Marshall, N., Fraser, G. W., et al. 1981, MNRAS, 197, 865

Watson, M. G., Ricketts, M. J., \& Griffiths, R. E. 1978, ApJ, 221, L69

Webb, N. A., Naylor, T., Ioannou, Z., Charles, P. A., \& Shahbaz, T. 2000, MNRAS, 317,528

White, N. E., \& Marshall, F. E. 1983, IAU Circ., 3806, 2

White, N. E., \& van Paradijs, J. 1996, ApJ, 473, L25

White, N. E., Parmar, A. N., Sztajno, M., et al. 1984, ApJ, 283, L9

Wijnands, R., \& Miller, J. M. 2002, ApJ, 564, 974

Wijnands, R., Miller, J. M., \& van der Klis, M. 2002, MNRAS, 331, 60

Wood, A., Smith, D. A., Marshall, F. E., \& Swank, J. 1999, IAU Circ., 7274, 1

Woods, P. M., Kouveliotou, C., Finger, M. H., et al. 2002, IAU Circ., 7856, 1

Wu, K., Soria, R., Campbell-Wilson, D., et al. 2002, ApJ, 565, 1161

Wu, J., Orosz, J. A., McClintock, J. E., et al. 2015, ApJ, 806, 92

Yamauchi, S., \& Nakamura, E. 2004, PASJ, 56, 803

Yang, Y. J., Kong, A. K. H., Russell, D. M., Lewis, F., \& Wijnands, R. 2012, MNRAS, 427, 2876

Yungelson, L. R., Lasota, J.-P., Nelemans, G., et al. 2006, A\&A, 454, 559

Zdziarski, A. A. 2014, MNRAS, 444, 1113

Zhang, S. N., Wilson, C. A., Harmon, B. A., et al. 1994, IAU Circ., 6046, 1 Zurita, C., Sánchez-Fernández, C., Casares, J., et al. 2002, MNRAS, 334, 999

Zurita, C., Casares, J., Hynes, R. I., et al. 2004, MNRAS, 352, 877

Zurita, C., Rodríguez, D., Rodríguez-Gil, P., et al. 2005, ATel, 383, 1

Zurita, C., Corral-Santana, J. M., \& Casares, J. 2015, MNRAS, 454, 3351

Życki, P. T., Done, C., \& Smith, D. A. 1999, MNRAS, 309, 561 


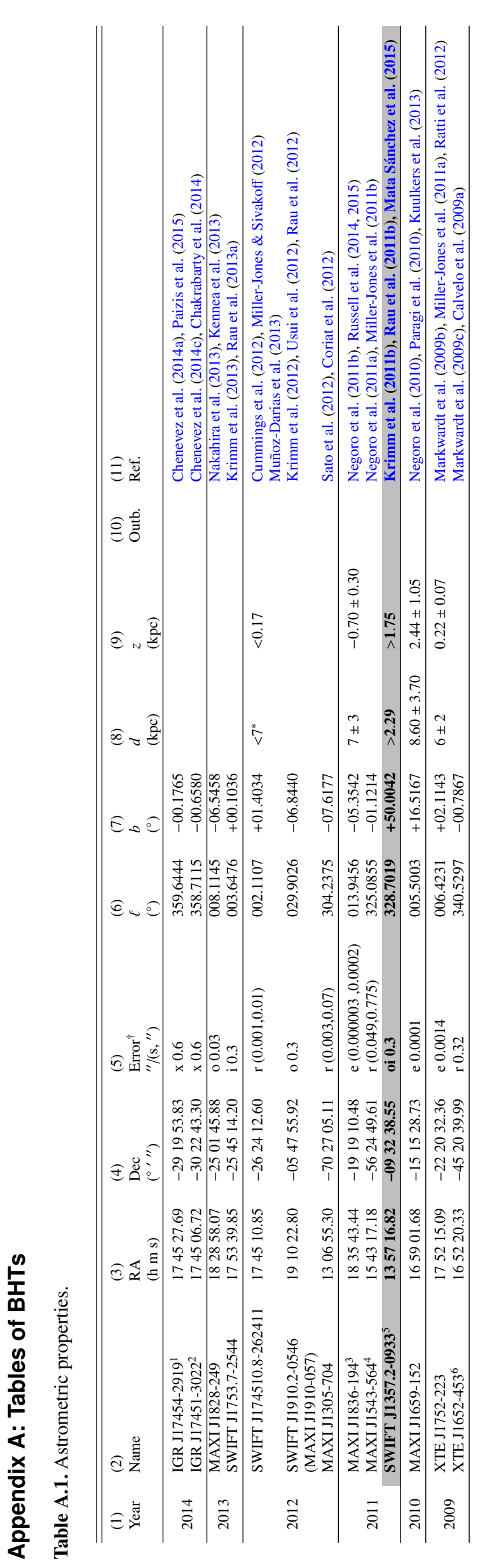

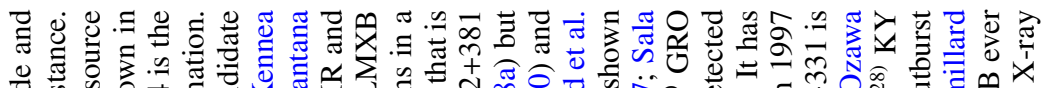

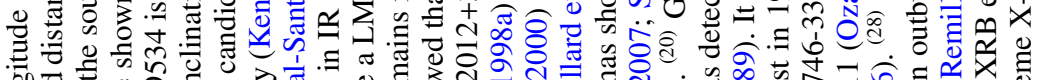

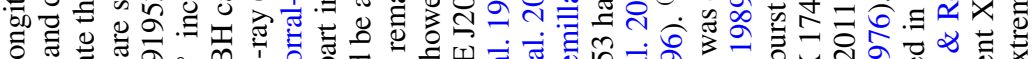

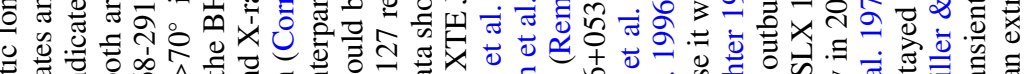

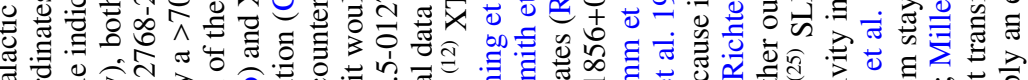

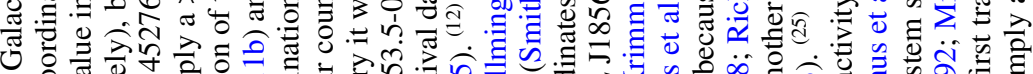

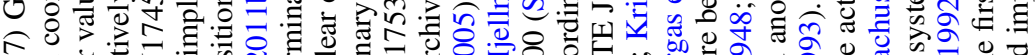

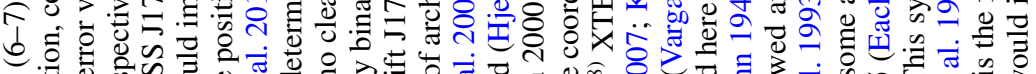

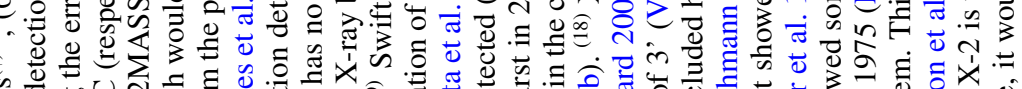

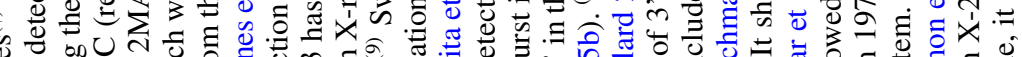

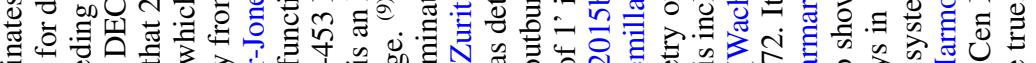

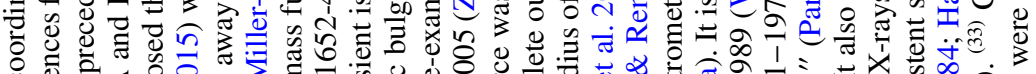
80 웡

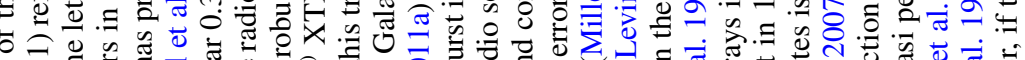

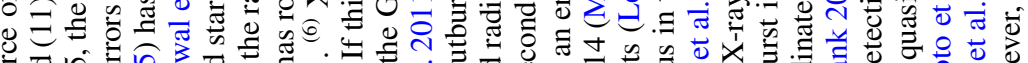

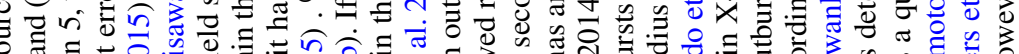

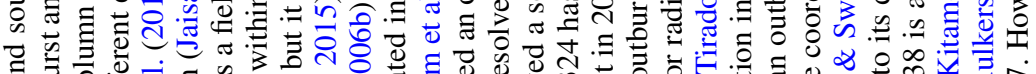

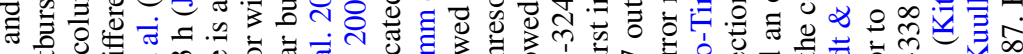

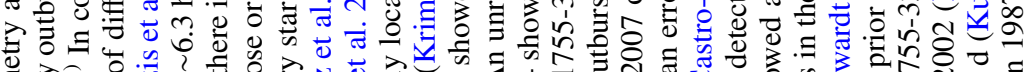
䛧

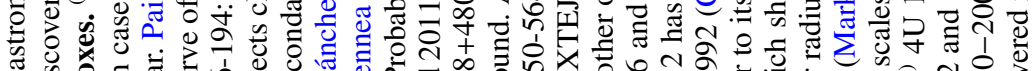

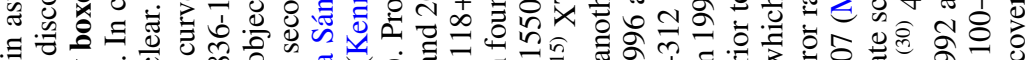

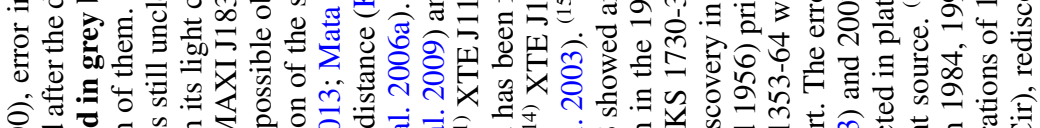

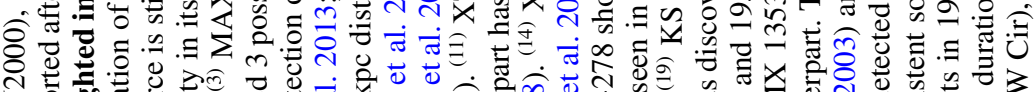

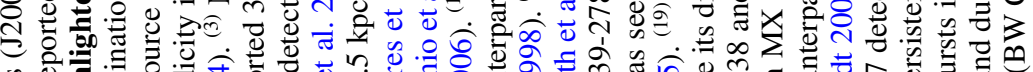

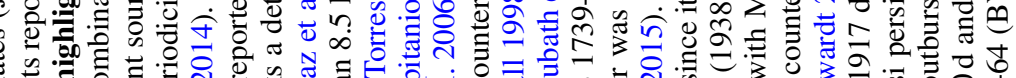

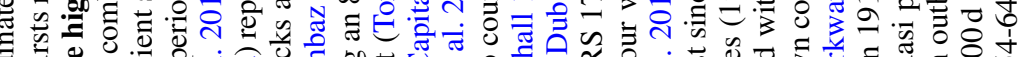

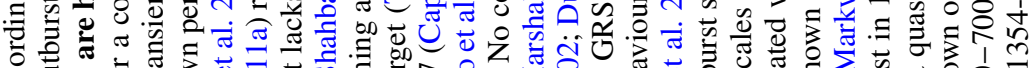

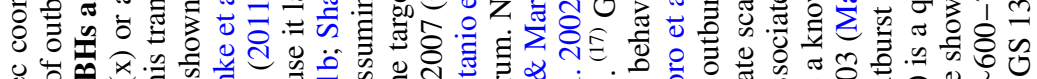

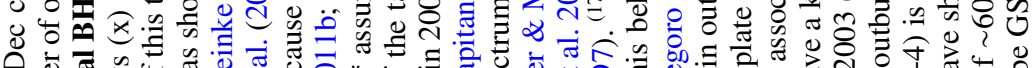

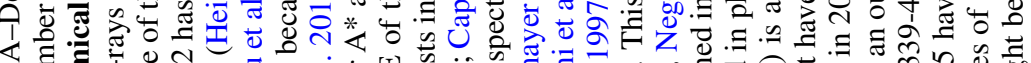

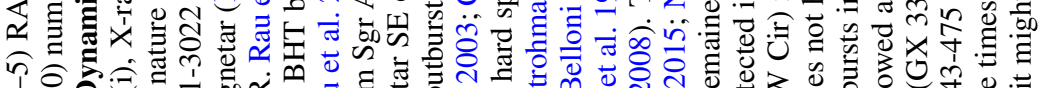
cô

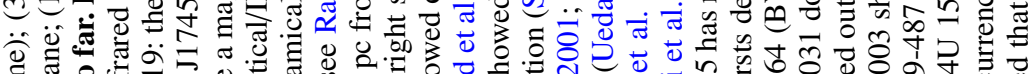

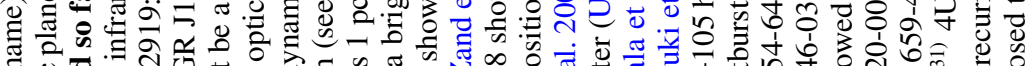

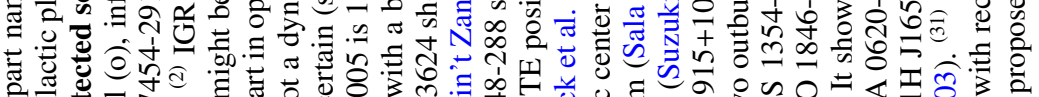

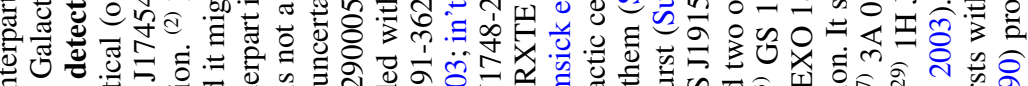
o 은

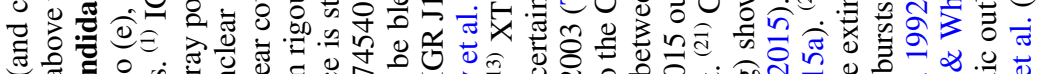

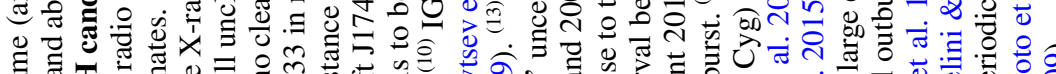

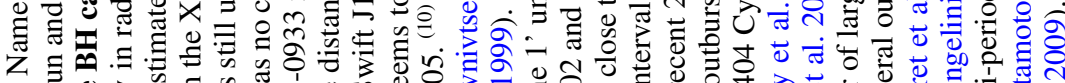

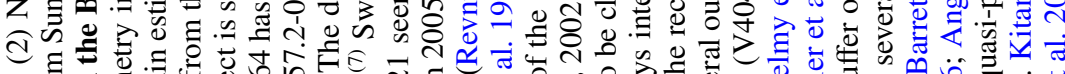

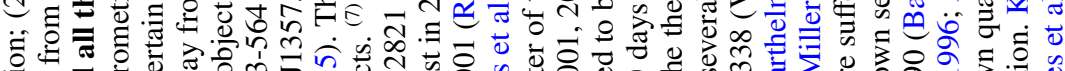

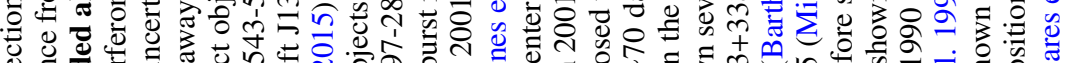

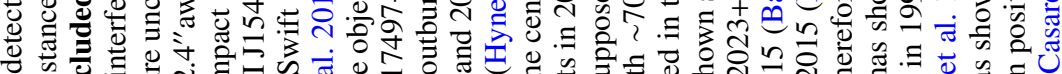
5

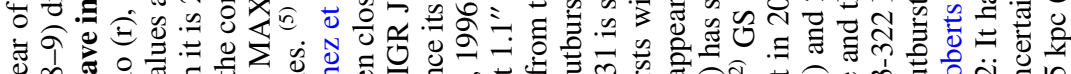

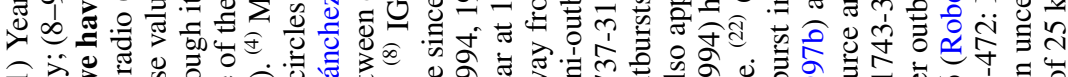

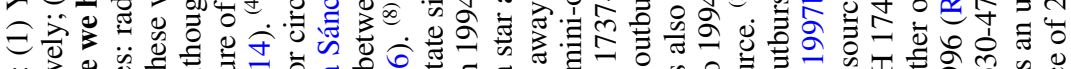

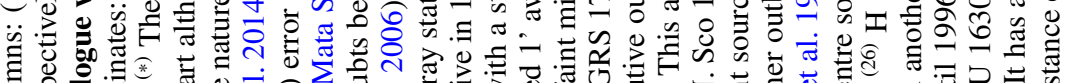

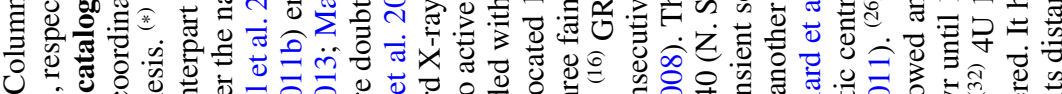

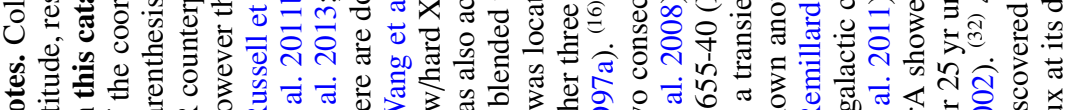

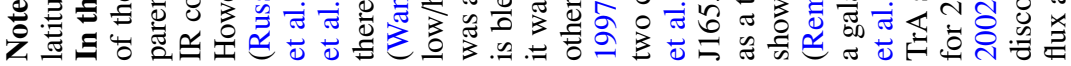




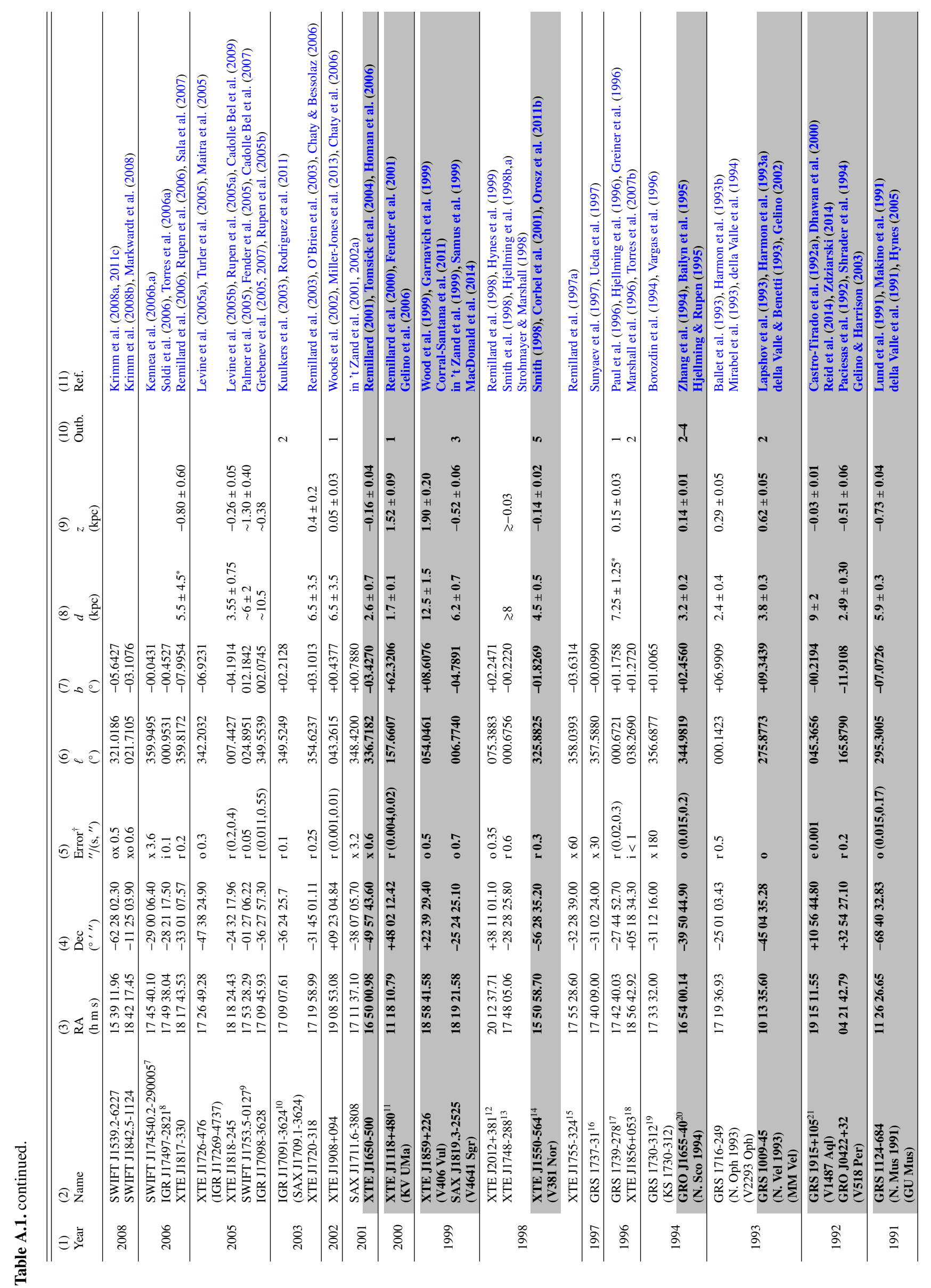


J. M. Corral-Santana et al.: BlackCAT

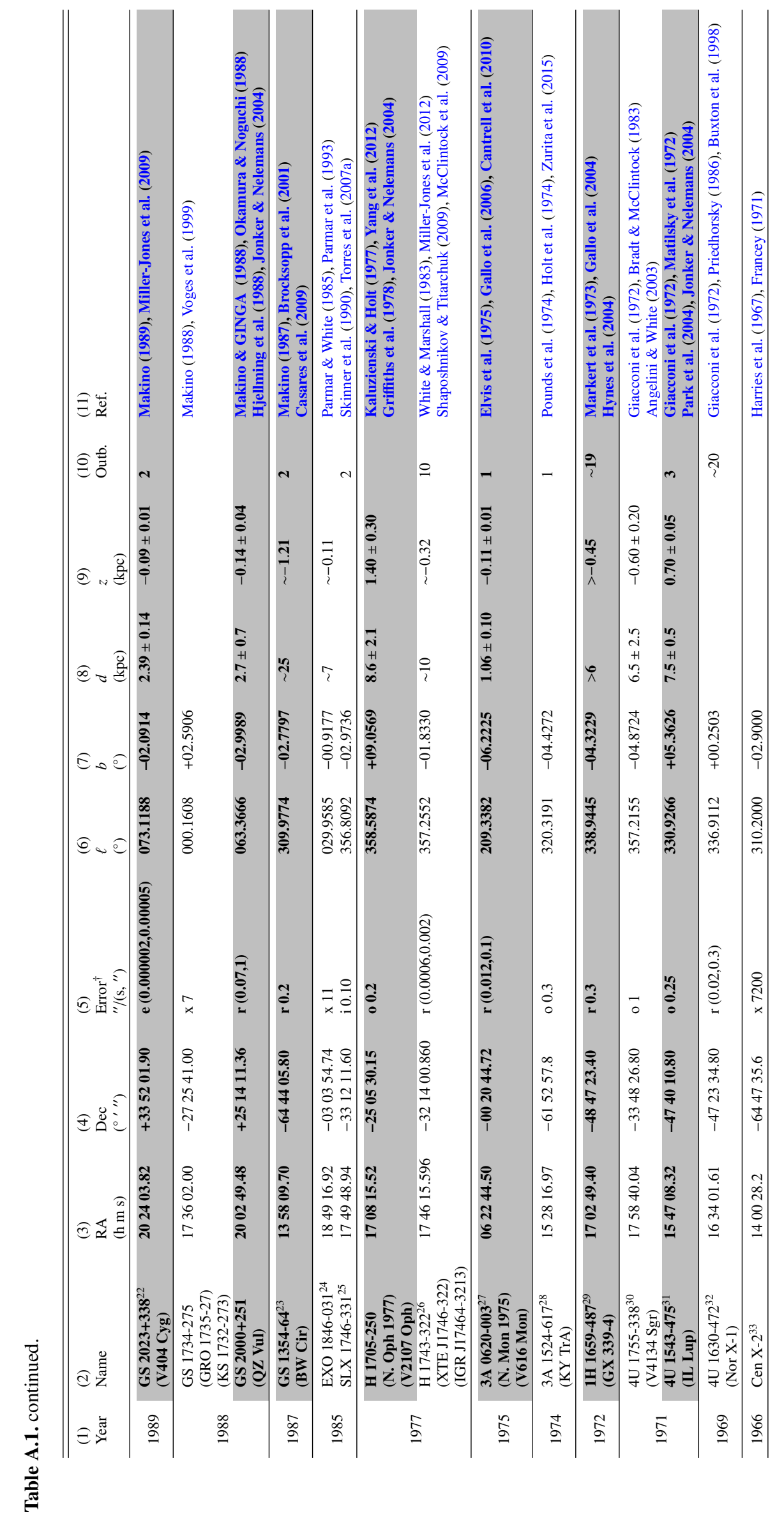




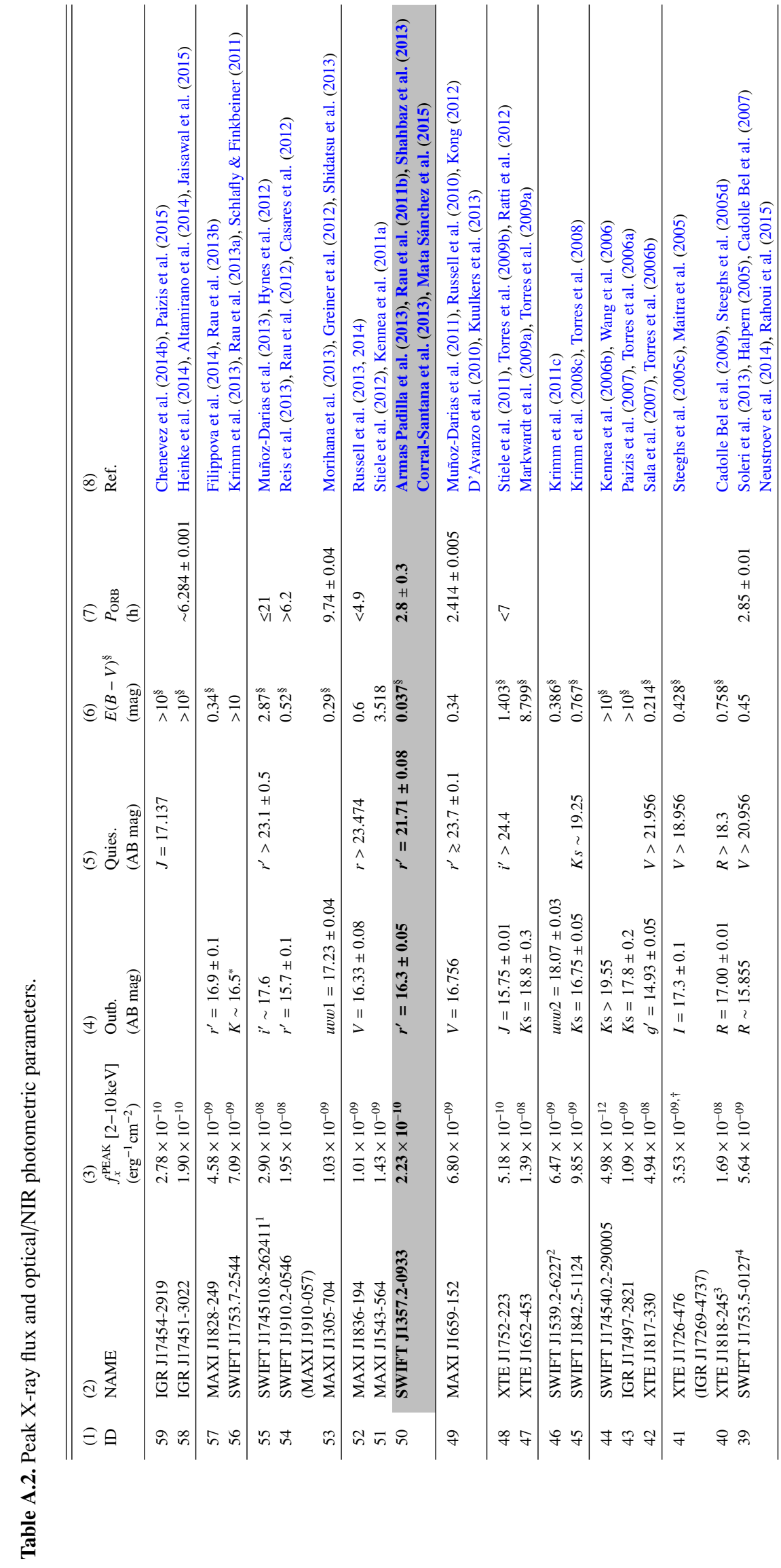

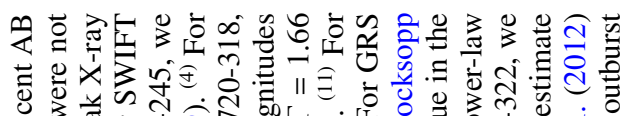

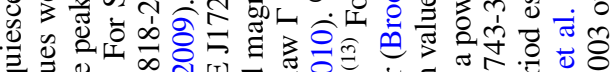

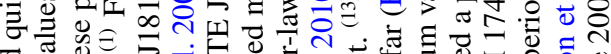

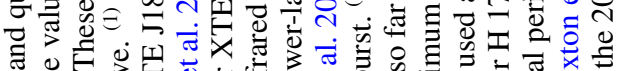

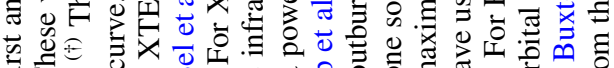

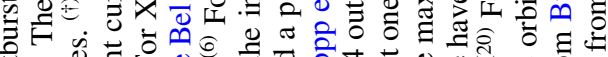

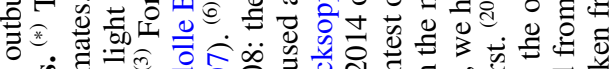

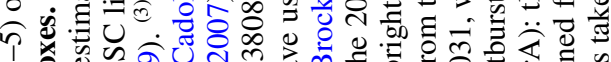

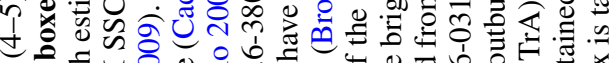

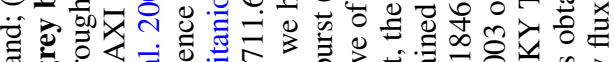

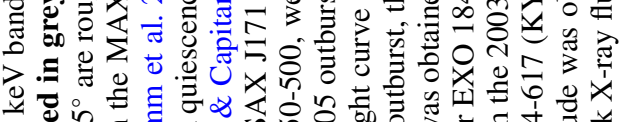

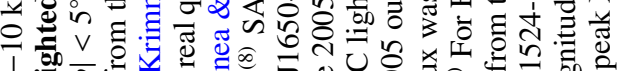

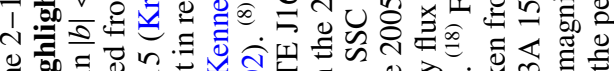

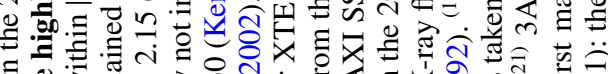

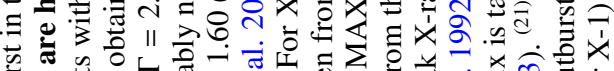

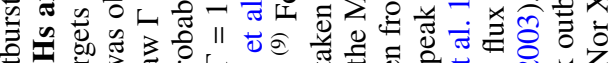

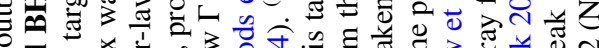

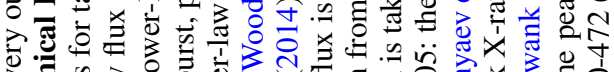

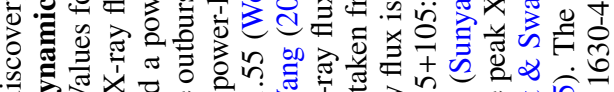

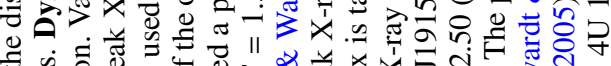

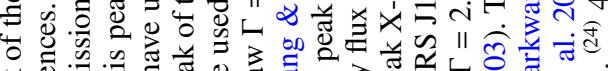

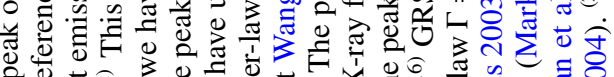

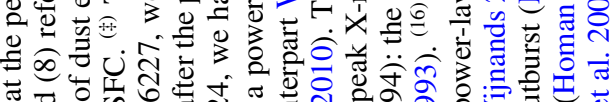

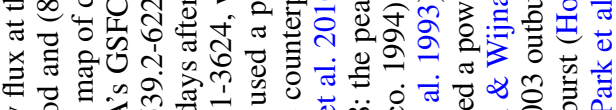

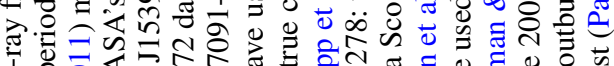

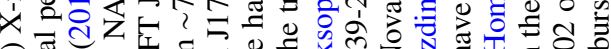

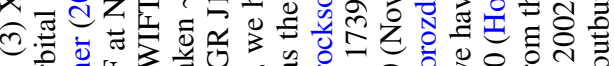

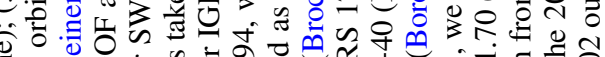

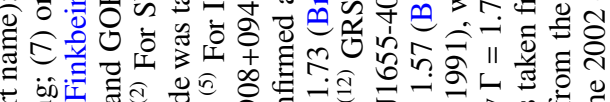

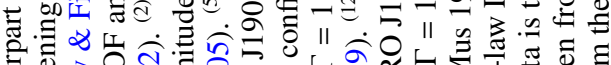

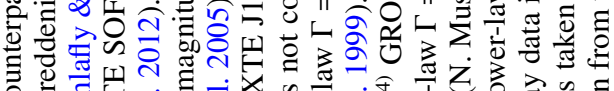

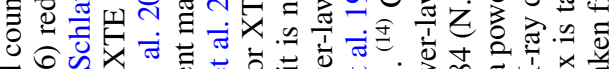

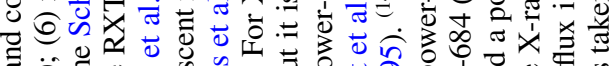

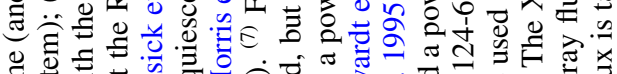

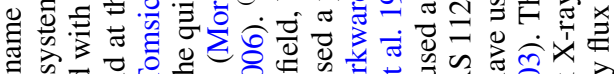

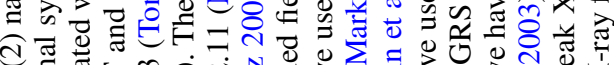

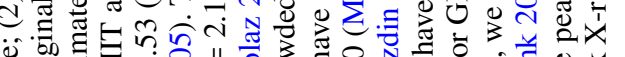

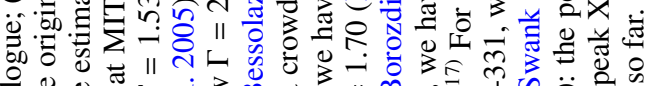

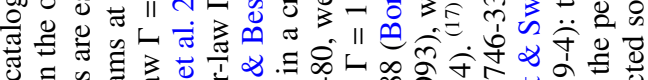

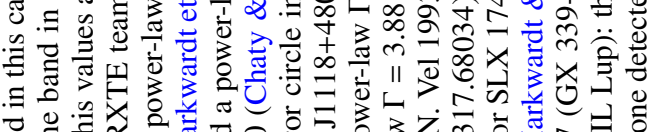

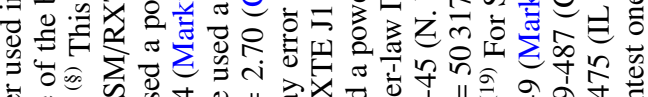

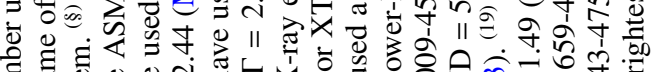

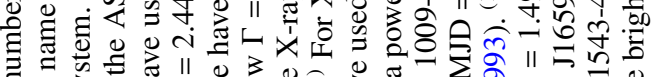

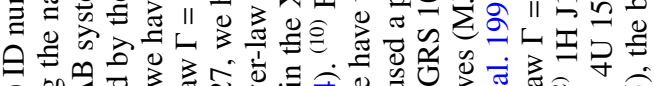

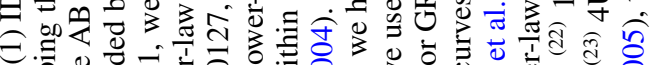

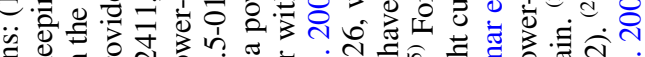

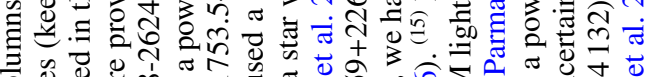

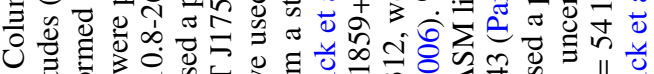

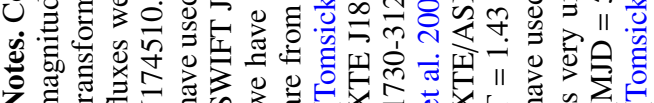




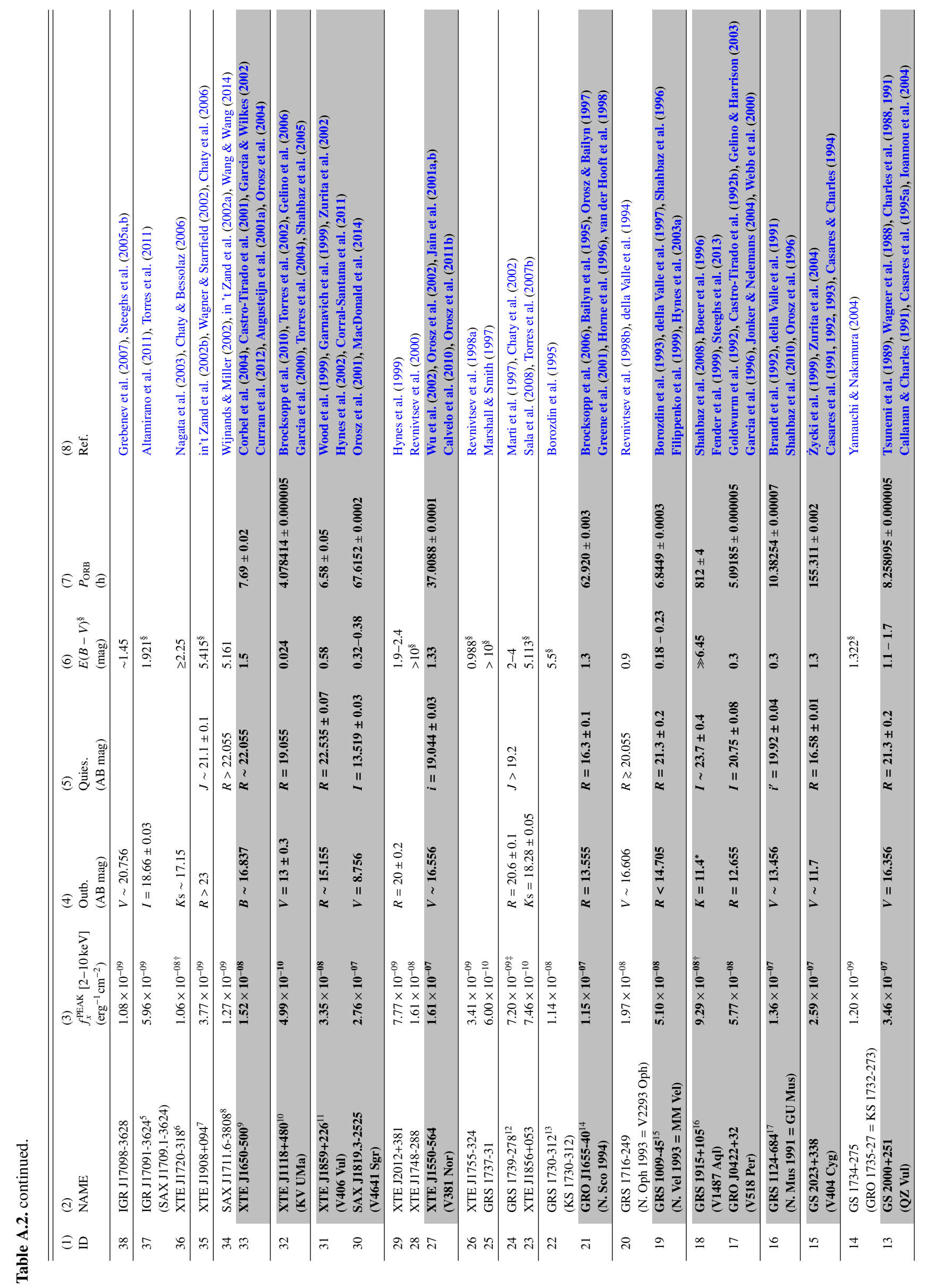


A\&A 587, A61 (2016)

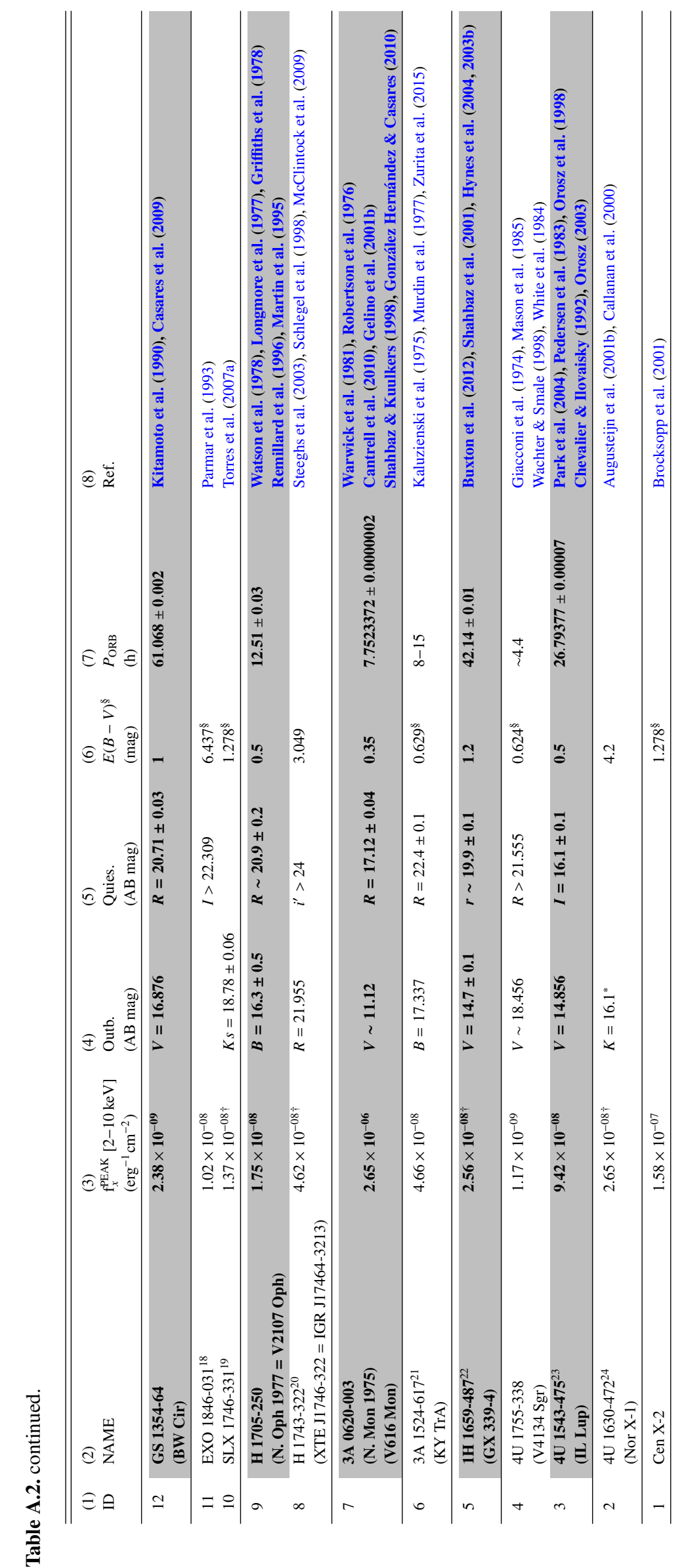




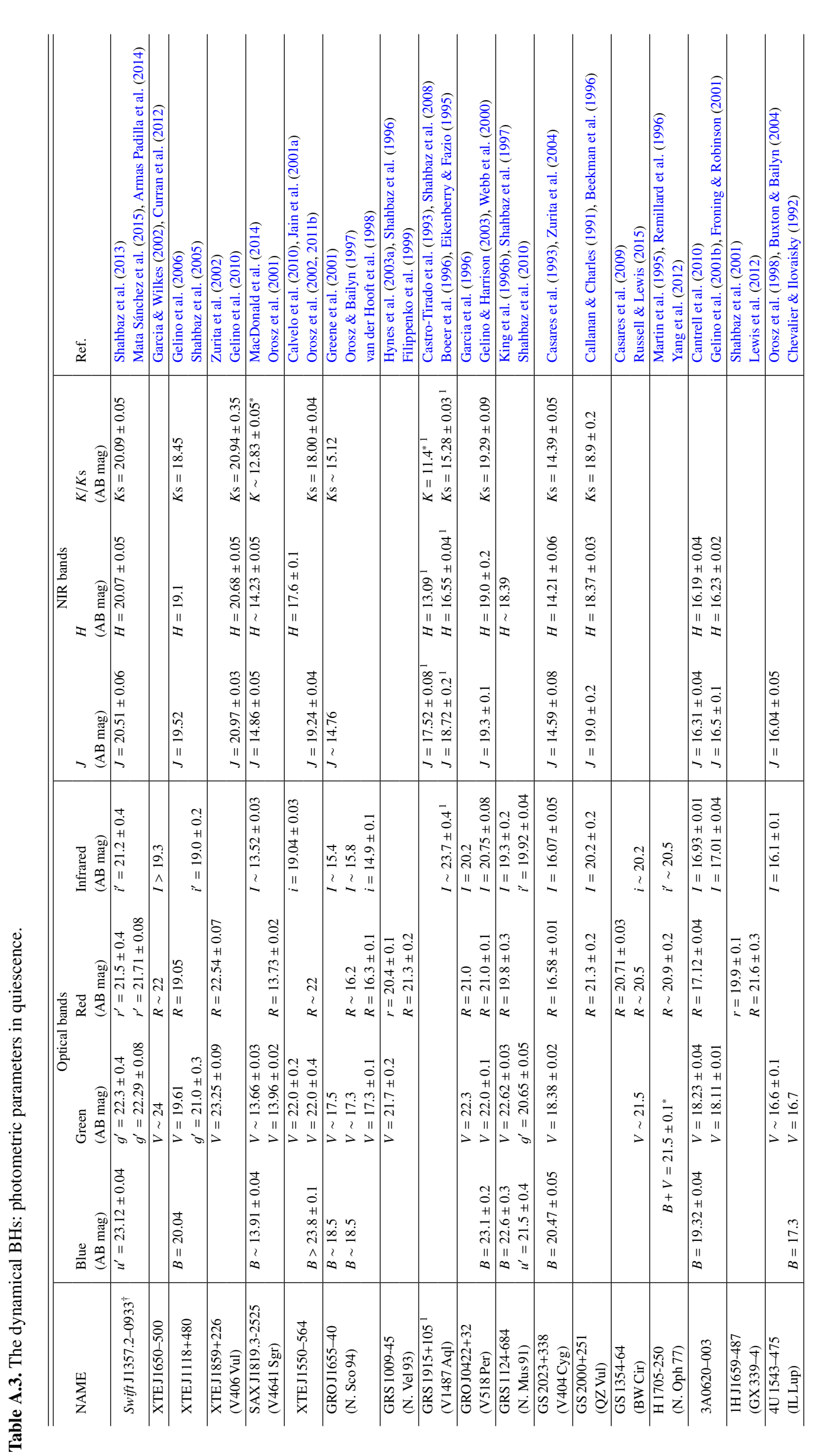

을 : 政 ô

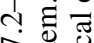
政高 赵 证 ois 긍

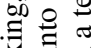
究

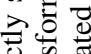

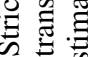
$E$ प्रे० 卷 大) z⿺ 一ํ. 政 要定 远 흘 훈 政害

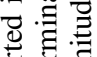

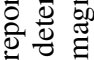
s. 등 施 踏

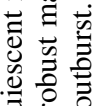
흥 它焉 氖言 这壳造 昰 o 요 용 范 읭 త్ 췅 S능 叫 ㄴㅇㅇ 空总声 Э. 毁

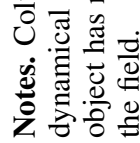




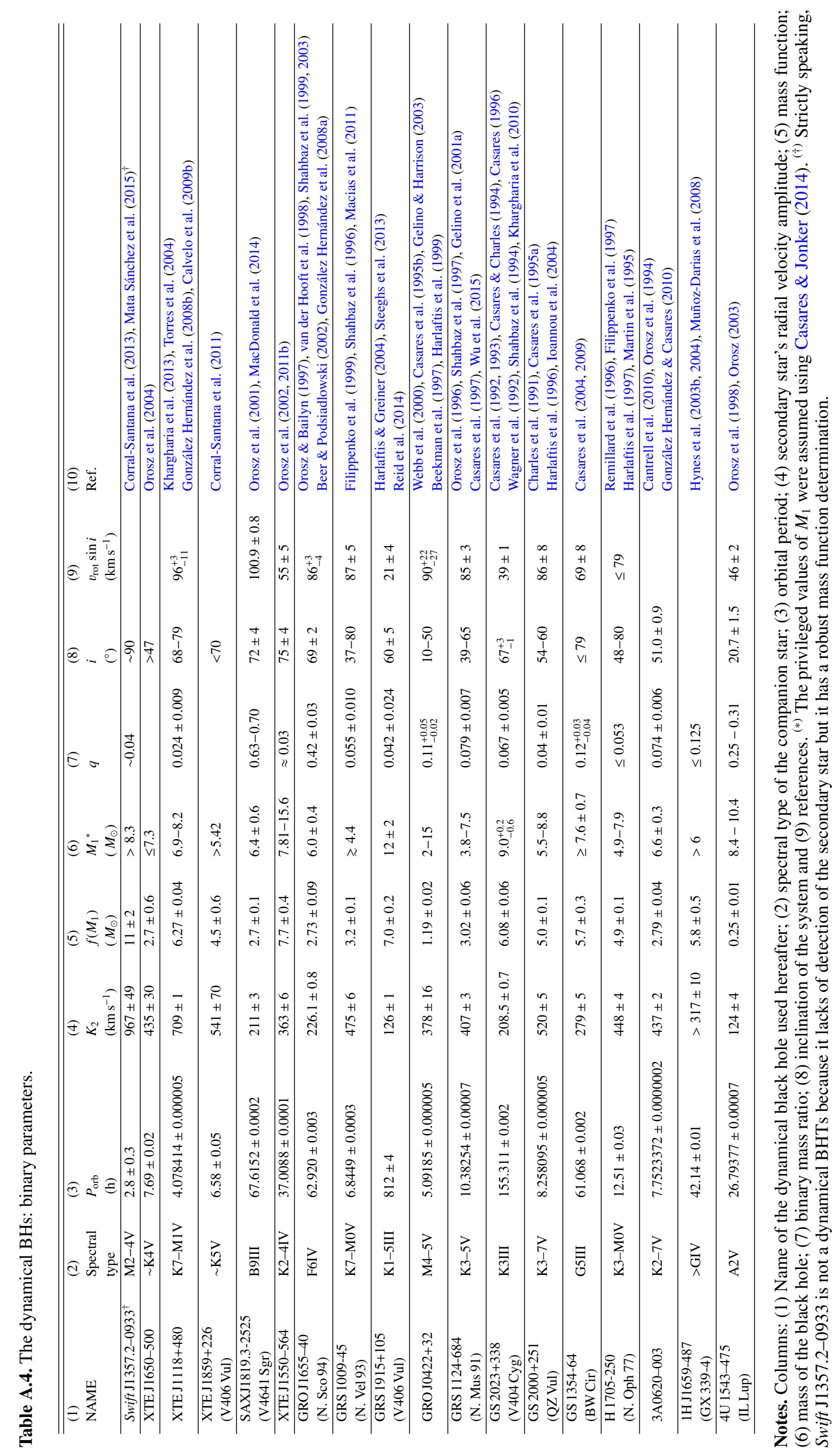

